\title{
Gradhiva
}

GRADHIV

Revue d'anthropologie et d'histoire des arts

$30 \mid 2019$

Précieux

\section{La fabrique du précieux}

\section{Marc Abélès et Franck Beuvier}

\section{OpenEdition}

Journals

Édition électronique

URL : http://journals.openedition.org/gradhiva/4253

DOI : $10.4000 /$ gradhiva.4253

ISSN : 1760-849X

\section{Éditeur}

Musée du quai Branly Jacques Chirac

\section{Édition imprimée}

Date de publication : 4 décembre 2019

Pagination : 10-31

ISBN : 978-2-35744-113-2

ISSN : 0764-8928

\section{Référence électronique}

Marc Abélès et Franck Beuvier, "La fabrique du précieux », Gradhiva [En ligne], 30 | 2019, mis en ligne le 31 mars 2021, consulté le 01 avril 2021. URL : http://journals.openedition.org/gradhiva/4253 ; DOI : https://doi.org/10.4000/gradhiva.4253 


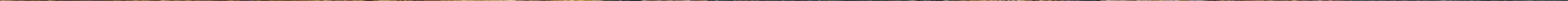


Introduction

La fabrique

du précieux

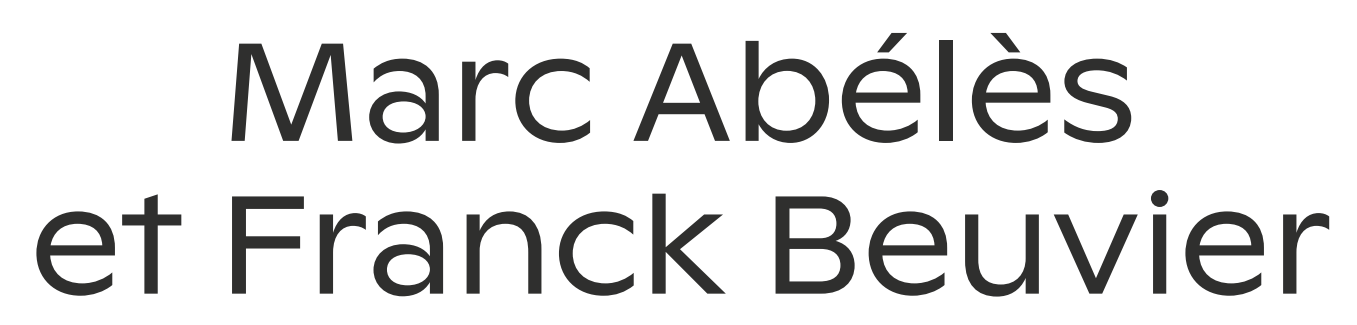




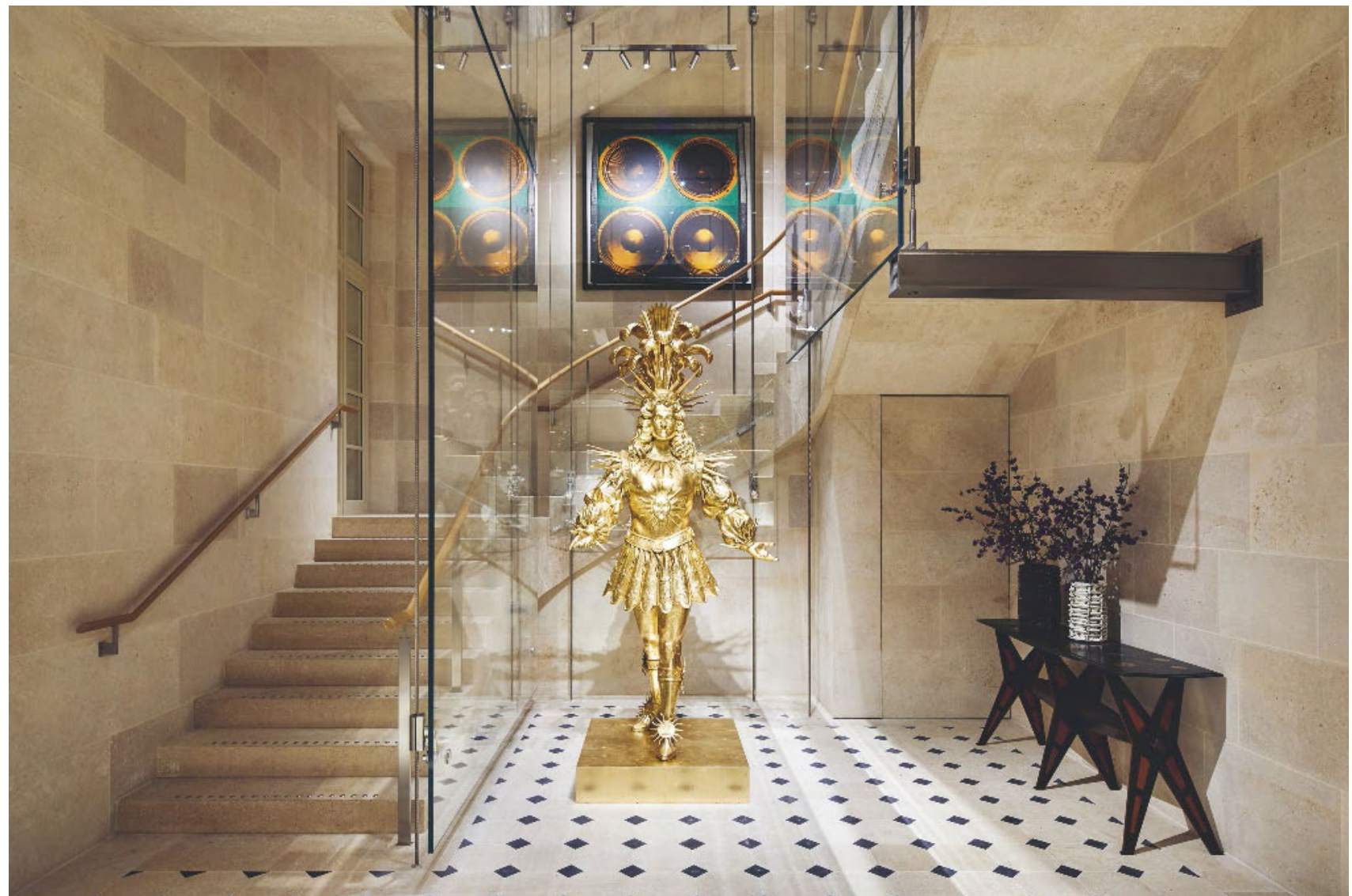

Boutique Louis Vuitton, rue du Faubourg-Saint-Honoré, Paris, 2017. Avec l'aimable autorisation de Louis Vuitton Malletier.

1. Malle chirurgicale (2009), présentée au musée Carnavalet en 2010, puis au Grand Palais en 2015

\section{ÉCHANGE ET TRANSMISSION}

Le 4 octobre 2017, Louis Vuitton renouera avec ses origines en inaugurant la Maison Louis Vuitton Vendôme, 2, place Vendôme. Un symbole sur une des places parisiennes les plus chargées d'histoire, qui marque le grand retour de la griffe dans le quartier où les articles de voyage, la mode et la joaillerie se côtoient depuis des siècles. C'est là, en 1854, que le jeune Louis Vuitton a ouvert son premier magasin et commencé l'écriture d'une histoire qui se poursuit encore aujourd'hui.

(Vuitton 2017:2).

16 janvier 2018, 10 heures. Nous venons de nous rejoindre place Vendôme, à l'anǵle de la rue SaintHonoré, devant la façade des anciens hôtels particuliers Heuzé de Vologer et Marquet de Bourgade, devenus officiellement la Maison Louis Vuitton Vendôme le 3 octobre 2017. Classée au titre des monuments historiques en 1933, la façade, édifiée par l'architecte du château de Versailles Jules Hardouin-Mansart à la fin du XVII ${ }^{\mathrm{e}}$ siècle, lors du premier aménagement de la place, se fond aujourd'hui en écrin. Les fenêtres hautes du rez-de-chaussée sont dans leur partie inférieure converties en baguiers, soulignnés par des cadres convexes revêtus de matière dorée et dévoilant quelques fines réalisations de joaillerie et de maroquinerie. Juste au-dessus, au centre des garde-corps en fer forgé du premier étage, rayonne un visage auréolé de l'astre solaire: l'emblème de Louis XIV, Roi-Soleil.
Avec les premiers clients, nous entrons par l'entrée principale rue Saint-Honoré. L'espace d'accueil, composite, offre principalement au regard accessoires et sacs à main. Sur les premiers présentoirs et les vitrines latérales, nous découvrons une sélection de portefeuilles, de lunettes, d'écrins riǵides, de souliers et de sacs, dont certains se remarquent dans leurs coffrets transparents. Aucune impression de trop-plein, chaque objet est soiǵneusement disposé. Quelques mètres plus loin, au seuil d'un ǵrand vestibule, trône une malle de grande dimension, rehaussée de motifs délicats de papillon sur la toile monogramme. Cette création de Damien Hirst ${ }^{1}$, protégée dans un coffre vitrine, parfait l'univers attendu de la collection et de la singularité. Sous nos pieds, un damier habille le sol, "un motif échiquier inspiré de la cathédrale Notre-Dame en pierre Croix-Huyart et pierre bleue» (ibid. : 20). Nous quittons un bref instant cette atmosphère pour nous présenter au personnel d'accueil. Notre venue a été signalée, et, au terme d'un mot de bienvenue, nous sommes invités à découvrir librement les lieux, munis d'un livret Louis Vuitton, d'un petit catalogue des artistes et des œuvres qui agrémentent les « univers» de la Maison.

La réunion des deux hôtels particuliers, formant ainsi le nouvel établissement, se veut un ambitieux projet de restauration. Le récit national attaché au lieu, à la place et à son oriǵine est souliǵné (ibid.: 3-5), tout comme le soin apporté à la rénovation du site. L'accent est mis sur la noblesse des savoir-faire mobilisés et des matériaux choisis pour rendre à l'édifice son âme et sa grandeur perdues, mais aussi sur la présence des œuvres d'art contemporain et le choix de procédés 


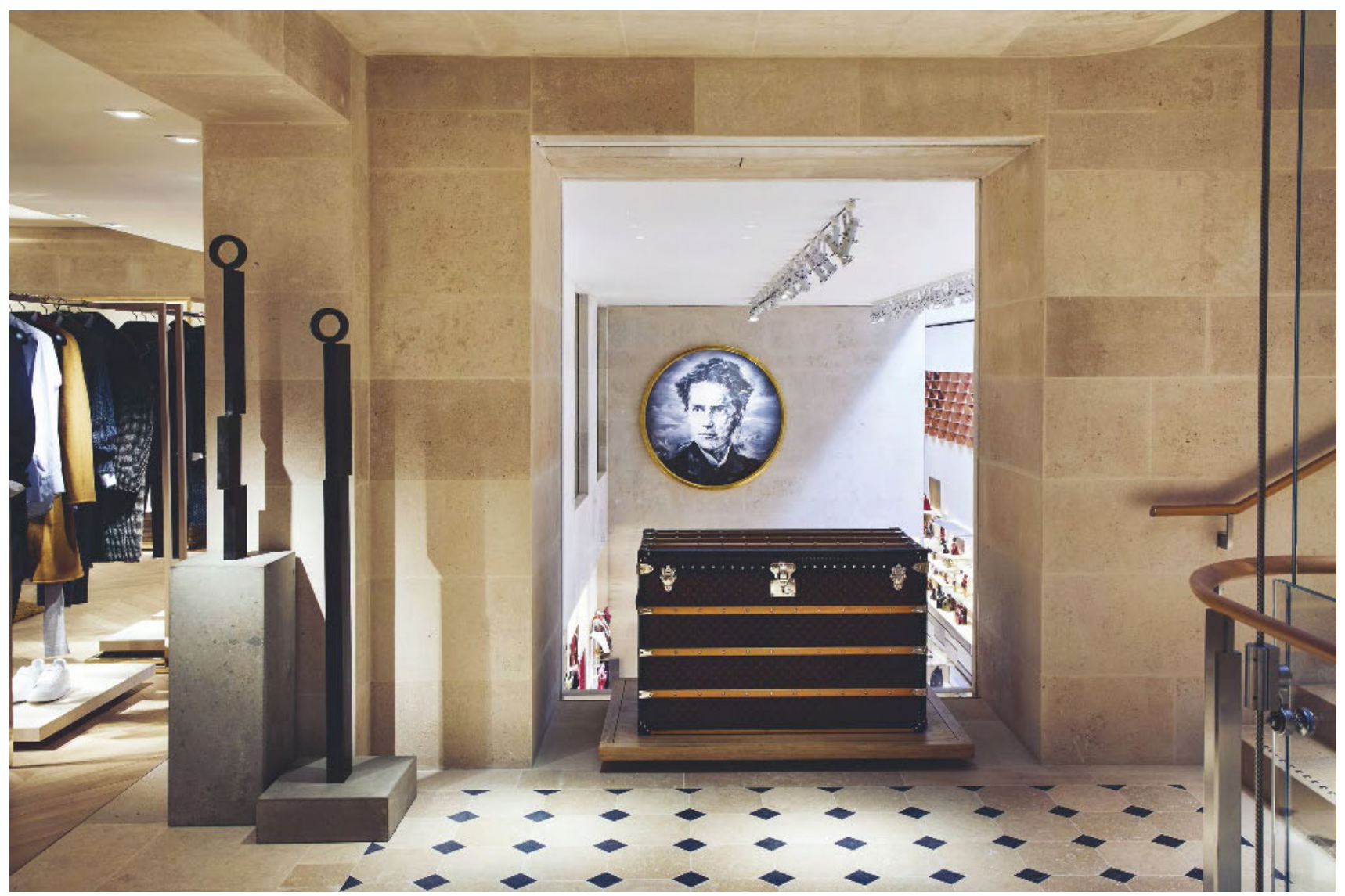

Boutique Louis Vuitton, rue du Faubourg-Saint-Honoré, Paris, 2017. Avec l'aimable autorisation de Louis Vuitton Malletier.

techniques permettant de créer un décor équilibré et soigné. Un accord, sinon un mariage entre l'artisanat du passé et l'art du présent, que l'on doit à «Louis Vuitton» et à l'architecte américain Peter Marino. Une «ode au savoir-faire et à la force de la simplicité», résume délicatement la brochure (ibid. : 17), la Maison Louis Vuitton rime avec poésie et émotions.

Avant de dévoiler l'architecture intérieure, le dossier de presse insiste sur les créations et les exclusivités proposées pour l'occasion : «Objets nomades» présentés pour la première fois en «édition permanente», «[fruits] de la rencontre unique entre la vision de célèbres designers internationaux et le savoir-faire artisanal de Louis Vuitton » (ibid. : 8); sacs en édition limitée dont le motif de la toile illustre une nouvelle série de transpositions de tableaux de «ǵrands maîtres» opérées par l'artiste new-yorkais Jeff Koons: Nymphéas de Claude Monet, Te nave nave fenua de Paul Gauguin ou Le Triomphe d'un poète de Nicolas Poussin, à découvrir uniquement place Vendôme; sac «Capucines», en hommage au premier magasin du fondateur; ou encore ces «trois parures de haute joaillerie», reflets de «la passion de la Maison pour l'artisanat d'exception et les plus belles pierres précieuses» (ibid. : 11). Pour accompagner les idylles de pierre, la marque présente sa "première malle joaillerie», une création aux dimensions remarquables et disponible sur commande.

Nous pénétrons ensuite les différents «univers» de la Maison. Au rez-de-chaussée, quatre espaces se dessinent, dédiés à la maroquinerie, aux parfums, puis aux «collections» d'horlogerie et de joaillerie, accessibles également par une entrée au 2, place
Vendôme. L'escalier central dessert l'entresol, aménagé pour accueillir les collections homme, et deux étages. Le premier étage est féminin. Signifié par la présence de plusieurs totems, un «cadre spacieux» s'offre à nous, «composé de parquet de Versailles, de toiles peintes et de murs parcheminés, ainsi que des œuvres signnées par Kimiko Fujimura et Annie Morris» (ibid. : 22). Sur le palier du deuxième, on découvre un large espace réunissant les articles de voyage et les «Objets nomades». Un univers onirique, fait de tableaux et de suspensions de grande dimension, qui se prolonge dans sa partie droite en une lonǵue table sur laquelle sont rassemblés les City Guides Louis Vuitton. Masqué par une imposante machine-outil ancienne, un poste de travail se profile, un atelier servant à personnaliser les objets, avec sur le plateau crayons, pinceaux et flacons d'encre de couleur. L'atelier de l'artisan d'art. Ici, malles, valises rigides, valises à roulettes, sacs de voyaǵe aux volumes variables composent les notes d'un hymne à la mobilité et à l'errance. «L'espace se dotera également d'un grand écran vidéo qui fera voyager la clientèle à travers les œuvres exposées à la Fondation Louis Vuitton. » (Ibid.) Un voyage parmi les œuvres, un voyage dans l'espaceterre, un voyage aussi dans le temps comme nous le rappelle par mille détails le lieu. «33 œuvres, 22 artistes, 6 continents» se confondent avec le monument pour procurer une jouissance intellectuelle et esthétique.

Sur ce même palier, deux autres accès sont fermés. Le premier, côté place Vendôme, ouvre sur un atelier et sur «l'Appartement». «Traditionnellement lieu réservé à la chambre du Maître de l'hôtel particulier, l'Appartement est accessible sur rendez-vous pour 
2. Suivant la typologie proposée par Daniel Fabre (2015)

3. Ce dossier est le fruit des échanges qui ont eu lieu danslecadredelajourne d'étude « La ronde (sacrée) d'étude "La ronde (sacrée)
des biens précieux », Collège d'études mondiales, chaire "Anthropologie globale du luxe », Fondation Maison dessciences de l'homme,

31 mai 2018. offrir aux grands clients une expérience exclusive. » (Vuitton 2017. : 23) OEuvres d'art, objets témoins de l'histoire dynastique et pièces uniques se découvrent dans le cadre d'un accueil discret et personnalisé. «L'Atelier Rare \& Exceptionnel» est l'atelier de haute couture, destiné aux «célébrités» et aux «clients les plus prestigieux », où tissus et matières se conjuguent pour former des «pièces de collection». Protégé, le second dessert par escalier une longue mansarde, l'atelier de haute joaillerie, où «pierres d'exception, aux couleurs et aux origines les plus rares » nourrissent l'imaginaire des artisans joailliers (ibid. : 24). On précise aussi que ces deux ateliers abritent un «savoir-faire ancestral». Ainsi, La Maison Louis Vuitton invite curieux, connaisseurs et clients à faire une expérience originale et intégrale, un voyage dans «l'au-delà de la valeur» (Abélès 2018: 10), tout entier imprégné de cette «virtuosité sémantique» propre au luxe (Appadurai 1986: 38). Pour ce faire, promoteurs et créateurs du lieu ont réuni quatre grandes instances de la mise en valeur et de la mise au secret: le trésor, le monument - associé au patrimoine - et l'art ${ }^{2}$, en jouant avec l'espace et le temps, avec ces amplificateurs de la valeur repérés par Nathalie Heinich (2017 : 258-268) : rareté, universalité, nouveauté et pérennité.

L'Essai sur le don de Marcel Mauss donne un coup de projecteur durable sur la question des biens précieux en anthropologie (2012 [1950] : 143-279). Point de départ d'une modélisation des principes de l'échange, ce texte dresse un portrait du précieux sur la base principalement d'une description croisée des systèmes codifiés de prestations à l'œuvre sur la côte nord-ouest des États-Unis et en Mélanésie. Placée sous le signe de l'émulation, la circulation des biens précieux fait intervenir des personnes morales et s'opère dans le cadre de ce que nous pouvons appeler des dispositifs: des situations empreintes de solennité qui mobilisent et mettent en scène différentes catégories sociales, des discours, des codes de conduite, des temps festifs, des temps rituels, des protocoles militaires, etc. Des ensembles hétérogènes, pour reprendre le mot de Michel Foucault (2001 [1975]), que Mauss nomme «systèmes de prestations totales ", dont la structure dépend de facteurs d'ordre politique, économique, juridique, moral, religieux et social. Seconde caractéristique relevée par l'auteur: la raison première de l'échange réside dans l'obligation absolue de rendre, en vertu d'une qualité très particulière du bien précieux, de l' «âme» donnée à la matière. Le précieux est par nature vivant, doté d'une «force» qui oblige. Réceptacle d'histoires individuelles et collectives, il est «habité», à l'abri des aléas de la relation contractuelle. Conjointement, le précieux est propriété, rendue tangible par un ensemble de liens formés avec le temps entre des individus et des lieux. Gage de stabilité et de continuité, il est, pour ainsi dire, valeur absolue.

Ce texte préfigure les approches ultérieures du bien précieux, où la définition de son statut, par opposition à d'autres types de biens, participe du développement croisé des champs économique et politique de l'anthropologie (Polanyi 1957; Meillassoux 1960; Bohannan et Bohannan 1968; Sahlins 1976 [1972]; Gregory 1982; Warnier 1985). La catégorie des marchandises de prix devient un point de repère à partir duquel se dessine une caractérisation des modes de production et de circulation, une typologie des biens échangés en dialogue avec les formes d'organisation et de hiérarchisation sociales. De là, le bien précieux, en tant que catégorie d'objets matériels et immatériels dotés d'un statut particulier et d'une valeur ajoutée, permet de penser non seulement les systèmes socioéconomiques et les modalités de l'échange, mais également les faits de domination, de distinction, de hiérarchisation et de reproduction. Don, contre-don, hors don, sauvegarde, contrat, marché, consommation..., L'Essai sur le don fournit à travers un large éventail de situations un vocabulaire propre à penser deux paradiǵmes complémentaires rappelés par Maurice Godelier: l'échange et la transmission (1996: 53).

Le bien précieux rime avec valeur. C'est l'une de ses propriétés constitutives, objet d'une attention soutenue, quel que soit le domaine considéré: valeur d'échange, valeur marchande, valeur esthétique, valeur morale, valeur historique, valeur spirituelle, valeur infinie lorsqu'il est déclaré unique ou consacré inaliénable. Au tournant des années 1990, un texte devenu classique, cité dans deux ouvrages de référence, va contribuer à rebattre les cartes et à déplacer durablement le champ de questionnement: Philosophie de l'argent de Georǵ Simmel (2014 [1911]). Deux perspectives ou deux usages de l'œuvre du sociologue allemand sont à relever: le premier orienté vers l'échange, fondement de la valeur; le second vers la conservation, où le bien, inaliénable, incarne une valeur absolue au principe de toute valeur relative. Dans un essai remarquable, Arjun Appadurai (1986) identifie le point nodal de la valeur dans l'échange, en s'attachant à la «vie sociale» des objets, à leur(s) itinéraire(s) et à leur(s) «carrière(s)» dans le processus d'assignation de la valeur. La «politique de valeur» se décline en «régimes de valeur», suivant des chemins tortueux, des désirs, des savoirs et des récits, un «esprit» changeant de la chose en somme, dans l'espace et dans le temps. Aux avatars de la circulation générateurs de valeur, Annette Weiner (1992) répond en souliǵnant un dispositif inverse, celui de la sauvegarde et de la transmission, qui non seulement porte en germe l'acte de valorisation, mais conditionne l'échange précisément. Dans chacun des cas, la destinée de biens coûteux et de biens inaliénables décide des voies empruntées. Les contributions réunies dans ce numéro ${ }^{3}$ invitent à privilégier l'entre-deux, à se situer aux confins de l'échanǵe et de la transmission. Systématiques, comparatives ou descriptives, elles nous parlent de signes - pour reprendre le mot de Jean Baudrillard (2015 [1968]) - aussi divers que l'article de luxe, la ǵriffe, la collection d'art primitif, le fétiche, le bien coutumier, la peluche du bébé, La Joconde de Léonard de Vinci, les exotica ou les biens en coquillage. Un ensemble au premier abord hétérogène, à moins de considérer le caractère irremplaçable de ces objets. A minima, ils partagent cette qualité d'être précieux, 
tous se situant ou se voulant au-delà de la valeur. Leur raison d'être commune. Ce statut ou cette bonne étoile qui les distingue brouille la liǵne de partage entre l'échange et la transmission en raison des efforts consentis pour s'assurer de leur pérennité, ou, à tout le moins, pour les inscrire dans une continuité. Si les articles de luxe oscillent entre «superfluité» et valeur cardinale (Abélès 2018: 35), ou si le doudou court le danger de perdre un jour son aura, ils ne s'éloignent jamais complètement de leur fétiche ontologie, car ils aspirent à être ou à devenir des «objets-personnes » (Heinich 1993).

En ce sens, l'orientation retenue ici laisse de côté les rapports établis en anthropologiie entre objet précieux et modèle économique. Non que la question soit épuisée, elle fut et demeure abondamment traitée, mais reste tributaire d'une autre, qui touche justement au «sans prix ». La première partie de la contribution de Julien Bondaz à ce numéro mérite pourtant que l'on évoque l'échange marchand et les hypothèses relatives au développement du capitalisme. Appadurai a salué l'intuition de Werner Sombart (1922) quant aux liens historiques entre luxe et capitalisme, ainsi que l'intérêt de sa démarche mêlant évolution des mœurs, attrait pour les marchandises de prix et émerǵence de la forme capitaliste de l'économie entre 1300 et 1800 . Marc Abélès a proposé une nouvelle lecture de cet ouvrage fondateur, en observant notamment ce double mouvement: non seulement l'industrie du luxe est tournée vers l'extérieur, "vers l'importation de matières premières parfois introuvables », mais elle témoigne aussi d'une «vocation fortement exportatrice» (Abélès 2018: 48 et 59). Ce goût des choses du monde et leur consommation constituent même un préalable à l'essor du capitalisme industriel, dira Chandra Mukerji (Appadurai 1986: 41). Julien Bondaz repère ici, à la suite de William Pietz (2005), la « conjonction historique du développement du capitalisme et de la découverte des objets maǵico-rituels africains », où la notion de fétiche devient une nouvelle «valeur sociale des objets ». Suivant l'argument, «caractères fétiche de la marchandise et marchand du fétiche» ne font qu'un. Et lorsque l'on regarde l'activité des marchands merciers au XVIII ${ }^{\mathrm{e}}$ siècle, qui conçoivent meubles et objets d'intérieur à partir de matières naturelles et de productions humaines, de biens étrangers aussi curieux que précieux, le caractère fétiche semble devenir une propriété du luxe avec l'essor de ce que Pierre Verlet désigne comme le premier marché des objets d'art (1958).

À l'appui des propositions avancées par nos collègues, nous allons davantage examiner l'enveloppe de la chose, sentir l'émanation qui fait précieux, tout en soulignant qu'il y a aura et aura en la matière, car les dispositifs propres à la conversion en précieux diffèrent. Provisoirement, nous nommons ces dispositifs particuliers «mises en sacré », sans présager de la pertinence de l'énoncé, mais pour se démarquer du vocabulaire de l'échanǵe. Plus précisément, la «mise en sacré» se décline en discours, postures, expériences, représentations, filiations, rapprochements, idéaux, chimères, événements, lieux, rites ou liturǵies.

Des opérations mêlées et codifiées, indexées au temps, qui relèvent d'une sphère bien particulière de la production du sens. En fonction de leur nature, les biens pressentis pour entrer au panthéon du précieux mobilisent différemment ces propriétés et leurs possibles combinaisons. Bien que la majorité des auteurs usent avec réserve de la notion de sacralité dans le cas présent, partons néanmoins de cette idée pour envisager la nature de nos objets-personnes. Mentionné dans plusieurs contributions, le luxe, miroir de la tension fluctuante entre valeur absolue et valeur relative, nous servira de fil conducteur.

\section{ATTACHEMENT ET IDOLÂTRIE}

Nathalie Heinich, dans un essai d'axiologie du précieux, nous incite à la prudence. Si la préciosité d'un objet tient spontanément à son caractère onéreux, l'indice est en réalité trompeur. L'objet précieux vise avant tout le «sans prix», en cherchant, au moins pour un temps, à endosser les habits moraux de l'incessibilité. Il se veut irremplaçable, raison d'être de sa valeur qualifiante. Insubstituable donc, quand il n'est pas tout simplement inaliénable, tels sont les traits distinctifs du précieux. «Au-delà de la mesure», avance encore l'auteur, nous rencontrons «l'attachement», suivant la voie empruntée par Thierry Bonnot (2014), et le «jugement de valeur». Une ou des formes d'attachement plus exactement, ancrées dans des histoires en évolution, qui témoignent de marques de vénération et de liens de dépendance. Le terme «attachement», employé dans l'article de Pierre Schneider pour signifier le caractère «symbolique» des exotica dans la Rome du ${ }^{\text {er }}$ siècle, traverse aussi la contribution de Brigitte Derlon et de Monique Jeudy-Ballini. Le traitement réservé aux marchandises de luxe, aux pièces de collection et aux biens en coquillage se dévoile au travers de mots et de procédures, de traits de dévouement qui se cristallisent dans des «projections imaginaires».

Retenons l'attachement comme fil d'Ariane, que paroles et actes laissent soupçonner, en considérant ces affinités. Louis Vuitton, qui s'exprime à la première personne, nous invite au voyage, à un déplacement tant réel que métaphorique, où le dépaysement, l'appel au passé et l'allégorie plastique font office de stimuli. Le voyage, dans son panorama sémantique, mobilise de nombreux registres, qui vont du trajet à la traversée, de la promenade à l'expédition, du parcours initiatique aux périodes de la vie, de la généalogie familiale au déplacement dans le temps, de la révélation chamanique au rêve. Le voyage, c'est faire ainsi l'expérience de passage(s) dans certaines conditions. Rentrer dans la Maison Vuitton, c'est tout à la fois être dans ou appartenir à une histoire du lieu et du pays, ressentir la présence du fondateur, partager avec lui des valeurs anciennes et un certain art de vivre, participer au rayonnement de l'excellence nationale qu'il a contribué à perpétrer, toucher sa grandeur et inscrire nos pas dans les siens, se rattacher à une continuité esthétique. Glorieuse et hyperbolique, la Maison Vuitton est un superlatif. La proposition de René Magritte s'accorde avec la nature des produits présentés : «Ceci n'est pas du luxe.» C'est autre chose et bien plus. C'est être 


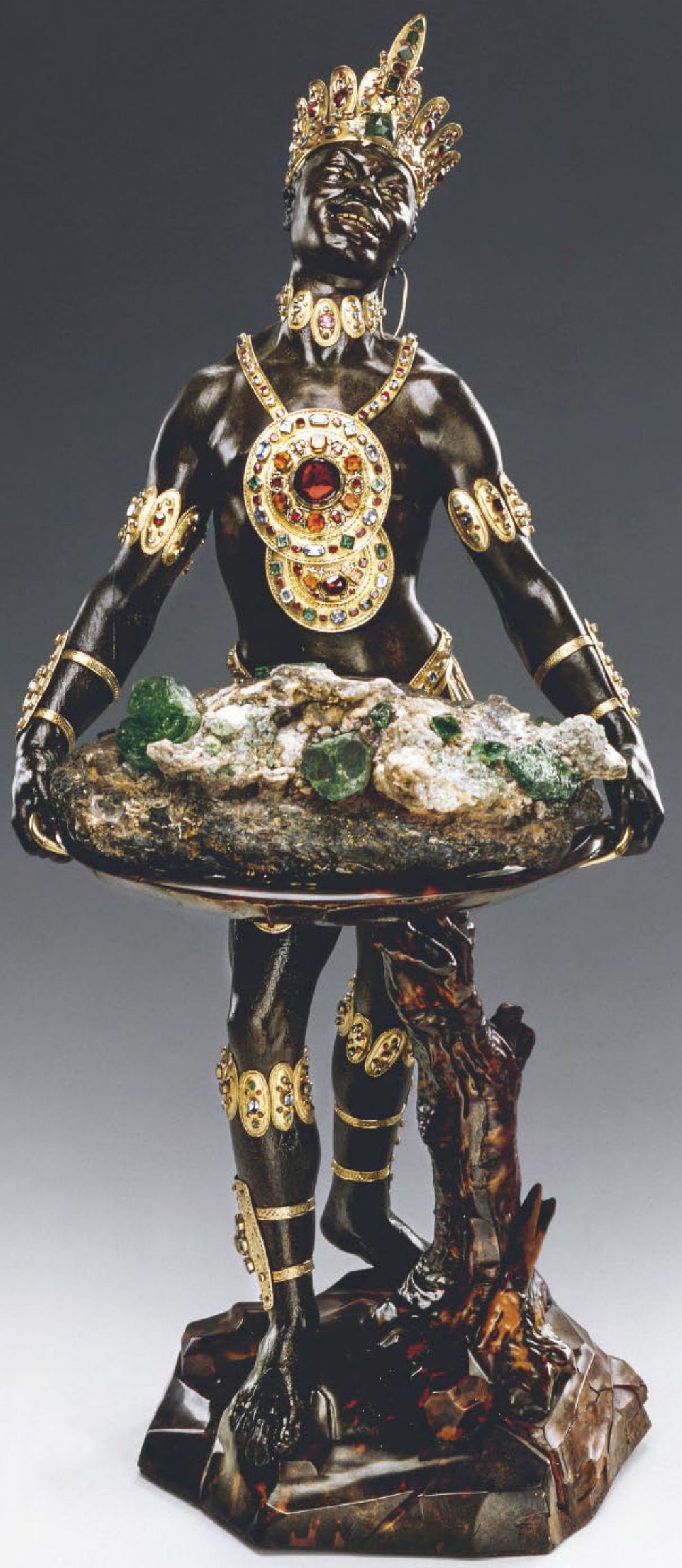

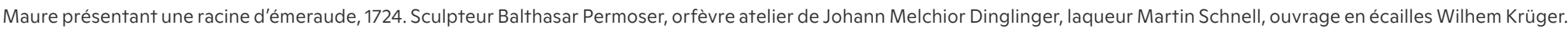
Dresde, Staatliche Kunstsammlungen. Photo ( BPK, Berlin, Dist. RMN-Grand Palais / image SKD. 
attaché à un tout justement, où rapport au temps et à l'exogène, mises en récit, en imaǵe, et en espace jouent un rôle essentiel. L'article de luxe, avancent Briǵitte Derlon et Monique Jeudy-Ballini, est «une image faite objet ». Une image à laquelle on tient.

Le merveilleux, en ce qu'il qualifie l'étonnement, l'admiration ou la maǵie engendrés par un artefact aux qualités exceptionnelles, est sans doute une des caractéristiques qui s'appliquent aux catégories d'objets retenues par les auteurs. Sous le régiime du principat d'Auguste, relate Pierre Schneider, Rome devient le lieu d'un véritable marché du luxe, où épices, gemmes, perles, écailles de tortue, soie, bois et matériaux bruts, venus de Chine, d'Arabie, de la Corne de l'Afrique, de l'Inde et du Sri Lanka, sont particulièrement recherchés. Les exotica figurent dans l'Histoire naturelle de Pline l'Ancien, où l'encyclopédiste s'attache à en restituer la place dans ce grand univers de la nature céleste et terrestre, et à en décrire les modes de consommation. En quoi sont-ils si singuliers? La préciosité de ces substances, matières, lames ou concrétions, appréciées conjointement à l'aune de leur valeur marchande et de la convoitise qu'elles suscitent, est liée à la fois à la renommée attachée à leur lieu d'origine, à des paramètres climatiques remarquables, et, de façon concomitante, à leur caractère minéral ou orǵanique. Elles sont en cela les fruits extraordinaires de la «Nature mère de toutes choses». Dans le cas des perles, l'attachement à la rareté tient au faible nombre de sites d'exploitation, aux conditions périlleuses de leur collecte et de leur acheminement, où le lointain se mue en désir teinté d'effroi. L'attachement prend inéluctablement racine dans ce que Pierre Schneider, à la suite de Pline, nomme la persuasio (conviction, croyance, opinion), nourrie par cet ailleurs et ses poignantes étrangetés. Les exotica convertis en biens de luxe rencontrent ici les œuvres d'art primitif et les biens en coquillage, dans un jeu de miroir de l'altérité radicale, qui se trouve suspendue entre deux infinis : l'un associé à l'idée du temps, l'autre à celle de distance. L'attachement façonne la part immatérielle de nos objets, nourrit leur légende ou révèle ce «double vital intangible» (voir Briǵitte Derlon et Monique Jeudy-Ballini dans ce numéro).

L'attachement est investissement affectif.

Véronique Dassié en a dévoilé les ressorts dans son ethnoǵraphie de l'intime, une vie secrète faite de souvenirs amassés au fil de visites et de voyages, de «petits riens » ou d'objets dépositaires de la mémoire individuelle ou familiale, qui peuplent l'intérieur du foyer et lui confèrent une âme (Dassié 2010). La nature de l'émotion suscitée selon ce qu'ils éveillent ou représentent, «indicateur de valeur» et «signal de préciosité », rappelle Nathalie Heinich, distinguerait à cet égard ce qui relève du souvenir, de l'héritage, du merveilleux, de la beauté, ou, pour le dire autrement, du passé, de la continuité, de l'ailleurs, de la pureté ou de la grandeur. Considérons la beauté, mère des valeurs du précieux, en poussant un peu plus l'argument. «La beauté déteste les idées. Elle se suffit à elle-même», écrivait Jean Cocteau (1959: 244), un indice qui révèle cette propriété avancée par Brigitte Derlon et Monique Jeudy-Ballini (2008) : «le caractère opératoire de la beauté», constitutif de ce lien familier que les collectionneurs d'art primitif nouent par exemple avec leurs pièces. Ce lien, observent-elles, naît d'un besoin de s'entourer d'objets, de la passion vécue à leur endroit et de leur indispensable présence. De façon concomitante, ces objets sont vivants. L'objet vaut pour une présence, selon le degré de vie qu'on lui accorde ou qu'on lui reconnaît, ou que l'on reconnaît en lui. L'expérience esthétique est comparable ici à l'expérience de la révélation mystique, constatent les auteurs. Cette présence particulière, dont le vécu émotionnel contribue à fortifier la voix, est celle du fétiche, reflet des figures de l'imaǵinaire colonial et missionnaire attaché à l'art primitif : le mystère qu'il incarne, ses vertus maǵiques, son lien avec les oriǵines, le monde de l'invisible qu'il rend tangible, etc. La collection ainsi réunie est en quelque façon monde et esprit. Ces pièces portent une histoire, témoignent du passé d'un ailleurs, terreau de l'humanité. Parmi les opérations de promotion ontologique à l'œuvre - pour reprendre la formule de Pierre Bourdieu (Bourdieu et Delsaut 1975) -, celle qui consiste à faire de la beauté un caractère apprécié à l'aune du passé prévaut, car le beau primitif se nourrit d'héritages, de généalogiies, de mythologiies. Dans le cas des collectionneurs, la beauté rime avec essence.

Alfred Gell a repris l'analyse des violences iconoclastes proposée par David Freedberg dans Le Pouvoir des images, en s'attardant sur les lacérations de la Vénus à son miroir de Velázquez, exposée à la National Gallery de Londres, infligées par l'artiste militante Mary Richardson en 1914. Un acte de déviance, qui, par contraste, révèle tout le «potentiel» de la représentation, un acte témoin de la volonté farouche de s'en détourner (Freedberg 1998 [1989] : 453, cité par Gell 2009 [1998] : 77). Pour Gell, le geste est politique: la jeune activiste a assimilé l'icône féminine de la mythologie à son égal contemporain, Emmeline Pankhurst, emprisonnée pour avoir défendu les droits civiques des femmes. Par ces lacérations, Vénus devient Emmeline, cet être remarquable en souffrance, suivant le rapport de ressemblance, d'identité, établi par Richardson (Gell 2009: 78). On pourrait, dans le même esprit, décrire le vandalisme qui accompagne la Révolution française, ces actes iconoclastes qui institutionnalisent pour un temps le rapport entre «le passé et l'émotion», en tant que gestes de dénégation d'un passé, et qui, paradoxalement, portent le désir moderne de la conservation (Fabre 2013: 29). À des fins de propagande, l'iconoclasme devient enseignement dans les situations de guerre, comme en témoiǵne Christina Kott (2013) au sujet de l'«Exposition d'œuvres d'art mutilées ou provenant des régions dévastées par l'ennemi au Petit Palais » en 1916. Dans chacune de ces circonstances, c'est bien le rapport d'identité entre l'objet et sa représentation qui est en jeu. «Un cas de sorcellerie des voults inversé», dit Gell au sujet de l'acte de Mary Richardson (Gell 2009: 78), une hérésie ressentie face au sentiment de la perte, invoque Daniel Fabre, une profanation commise avec ces œuvres mutilées de guerre, décrit Kott, où les restes des corps représentés choquent l'opinion tant leur présence martyrisée aggrave encore le bilan humain du conflit. Des vestiges meurtris, victimes de la vérité crue des combats. 


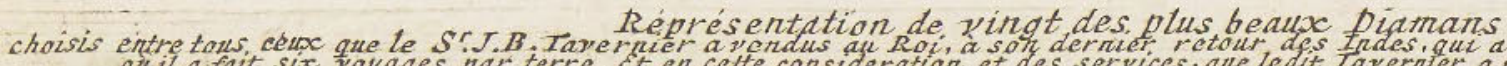

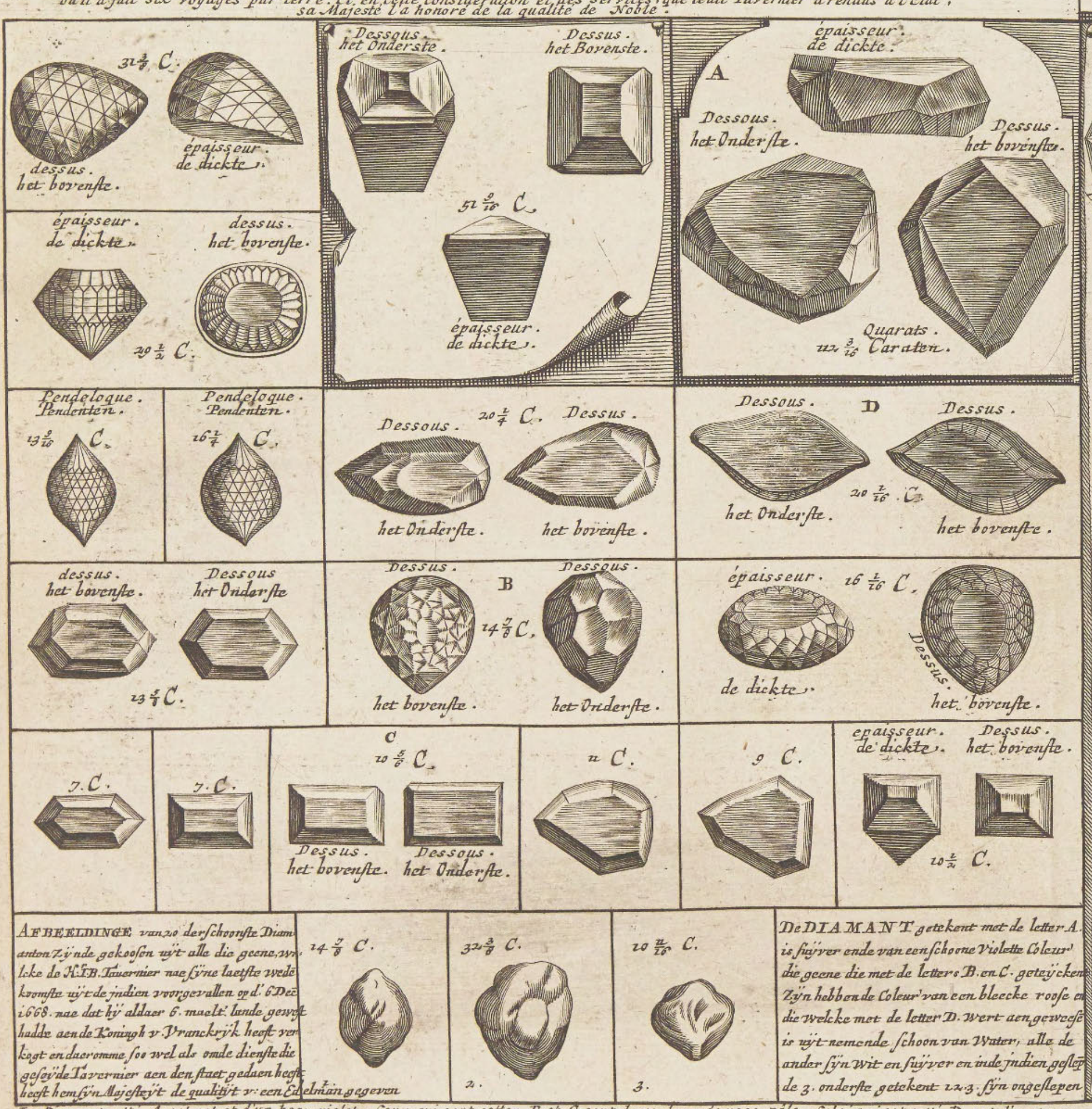

Iluit Diama

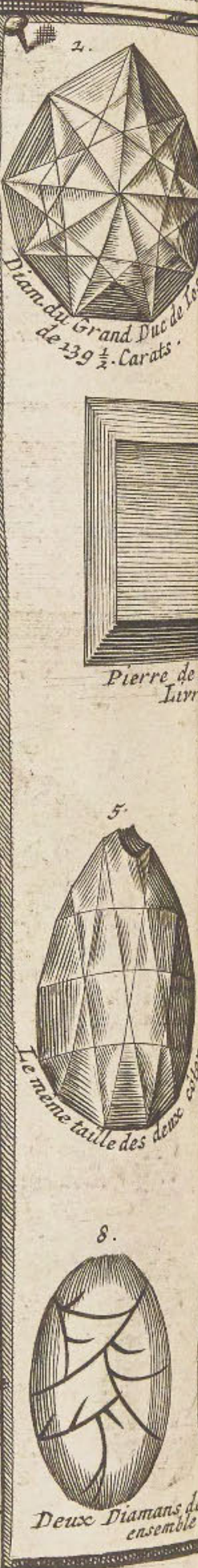

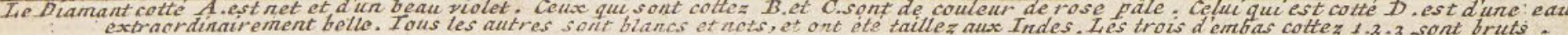



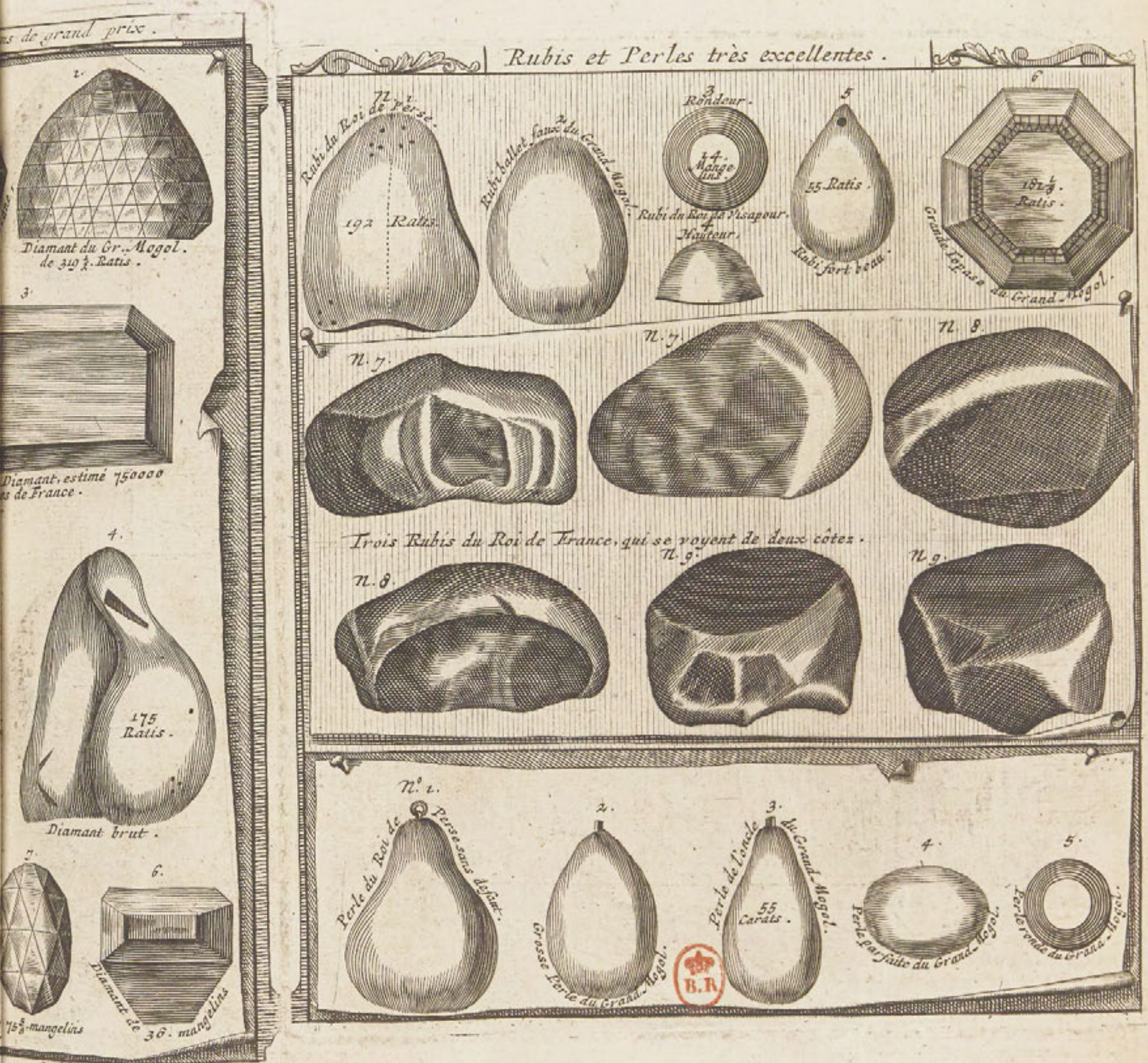
4. « Volez, voguez, voyagez - Louis Vuitton », Paris, Grand Palais (5 décembre 201521 février 2016).
Sans anticiper sur notre troisième argument, sur les liens de voisinage qui prévalent entre les objets précieux et les personnes, il est utile de reprendre la théorie de l'idolâtrie adoptée par Gell: un parti pris qui lui permet d'investir le culte des images sans donner crédit à l'expérience esthétique, en posant sur un pied d'égalité trouble religieux et trouble esthétique (Gell 2009: 120). En réalité, la malle Vuitton, le vase murrhin cher à la société romaine du $\mathrm{I}^{\mathrm{er}}$ siècle, l'objet de collection, le fétiche africain, La Joconde ou le doudou sont des idoles qui ne disent pas leur nom. L'émotion ressentie à leur endroit, soutient Gell, aurait été requalifiée au cours de l'histoire, suivant les cadres de référence nés du scepticisme reliǵieux des Lumières et de l'avènement de la pensée scientifique. «On peut donc naturellement en conclure que la distinction entre le beau et le sacré, entre l'expérience religieuse et l'expérience esthétique, résulte d'une vision biaisée [...].» (Ibid.: 121) Le point d'orgue de l'approche, pressenti avec l'attention portée à l'iconoclasme, est la faculté mimétique. «Sources» et «cibles d'agentivité sociale», les images sont enlacées dans des expériences et des usages particuliers. Les manifestations de l'idolâtrie - soin, rituel ou protocole - révèlent combien «les imaǵes sont [...] traitées comme des personnes» (ibid.: 119). L'idolâtrie serait finalement le sort réservé aux objets ordonnés personnes. Cette notion est retenue par Pierre Schneider pour rendre compte des comportements malséants décrits par Pline sous le terme insania ("déraison»). Signnificatifs à cet égard sont les agissements inspirés par les vases murrhins: bords rongés par son détenteur, une dégradation-personnalisation qui ne faisait que renforcer l'unicité et la valeur du vase, désormais lié à son adorateur; ou morceaux d'une coupe cassée entreposée dans une urne funéraire convertie en reliquaire. Un deliciae devenu perte inestimable, élevé au rang de trace. Dans le même esprit, pensons aussi aux délicatesses du collectionneur.

\section{OBJET ET PERSONNE}

$\mathrm{Au}$ 4, place Vendôme, lorsque nous atteiǵnons le palier situé à l'entresol, le regard est invité à associer deux éléments : une malle de grande dimension au premier plan, reposant sur un socle en bois, un presqueobjet de collection, et, au second plan, un portrait de «Louis Vuitton jeune», réalisé par le peintre chinois Yan Pei-Ming et présenté pour la première fois au Grand Palais en 20154 . L'exposition s'ouvrait par une correspondance assez similaire établie entre le portrait $\mathrm{du}$ fondateur et deux malles : l'une, en toile monogramme, datée de 1906, et l'autre, plus petite, conçue pour l'événement, faisant le lien entre le passé et le présent. À l'entresol de la place Vendôme, l'emplacement choisi pour présenter cette malle imposante laisse deviner une ancienne ouverture à double porte. La mise en communication des espaces crée une profondeur inattendue et conduit le regard vers le pan de mur sur lequel est fixé le tableau. Entre les deux, entre la malle et le portrait, un vide, qui laisse voir l'univers maroquinerie du rez-de-chaussée. Cette apparition, survenue sur le palier, nous renvoie aux portraits illustres qui veillent sur les montées d'escalier. L'effet de présence, du créateur et de sa création, est accentué par un embrayeur constamment mobilisé lorsque nous posons une question: "Monsieur Vuitton a souhaité que...» ou «Monsieur Vuitton a voulu que...». On ne perpétue pas seulement sa mémoire, on cultive sa présence. La Maison Vuitton place Vendôme prend ainsi des allures de maison de famille.

La malle, comme la plupart des objets précieux décrits dans ce numéro, relève d'une catégorie que Nathalie Heinich a nommée «objets-personnes» (1993, et infra.). Peu ou prou, ils répondent au critère d'«insubstituabilité qui caractérise le réǵime des personnes» (ibid.: 32). Tous partagent quelque chose en commun avec la relique, le fétiche ou l'objet d'art, en ce qu'ils sont particuliers, irremplaçables, et dotés d'une vie propre. Partons de la proposition avancée: «Il est trois façons pour un objet de posséder les propriétés d'une personne: premièrement, en tant qu'il agit comme une personne $[\ldots]$; deuxièmement, en tant qu'il a appartenu à une personne $[. .$.$] ; troisièmement, en tant$ qu'il est traité comme une personne [...].» (Ibid. : 27) Ces traits, qui renvoient successivement au fétiche, à la relique et à l'œuvre d'art, ne sont pas exclusifs les uns des autres, mais permettent de cerner une tendance dans chacun des cas. Typiquement, la préciosité, note Nathalie Heinich, se loge dans «la multiplicité des états de la chose», «une conséquence et une cause de l'extrême valeur qui lui est attribuée» (voir Heinich dans ce numéro).

De quel objet-personne se rapproche notre malle? Chez Louis Vuitton, Monique Jeudy-Ballini décrit ce que les vendeuses appellent les «vrais clients» (2000). Les «vrais clients» sont les acquéreurs de malles, de «rigides» selon le terme consacré, un produit qui représente le métier et l'ingéniosité du fondateur de la marque. Les malles incarnent en cela le «vrai, la Marque», la «Marque de la Marque», ou encore «l'âme de la Marque». Parmi les biens de luxe proposés, céder un «rigide» revêt un enjeu particulier. La vente s'apparente à un «événement», où la présentation du produit se mue en découverte doublée d'une expertise ayant valeur d'une authentification matérielle. Au terme du processus, qui plus est, on garde trace de l'échangée. On singularise l'acquéreur en établissant une presquefiche d'identité qui relie le bien et son destinataire (ibid. : 4). La filiation, ou le rapport d'identité établi entre la malle et son fondateur, s'étend aux acquéreurs de ce produit, désormais distingúés des clients ordinaires. Le vrai client est celui qui se voit rattaché aux origines. Qu'est-ce qu'acquérir une malle, sinon posséder un bout de l'œuvre d'un "grand singulier» (Heinich 1991 et 1993)? Le dossier de presse en précise le profil: «Devant rivaliser avec 339 autres malletiers travaillant à Paris [...], Louis Vuitton innove [...] et en 1854, il présente une malle plate révolutionnaire [...].» (Vuitton 2017: 3) Le génie de Louis Vuitton réside dans cette performance, point de départ de sa renommée, que lui assurera l'impératrice Eugénie (ibid.).

La malle n'est pas un fétiche. Presque œuvre d'art, elle est d'abord une relique. Objets de collection, les malles furent célébrées en tant qu' « œuvres historiques 
et actuelles» au Grand Palais en 2015. L'exposition «Volez, voguez, voyaǵez - Louis Vuitton » faisait ainsi la part belle à la richesse de la gamme développée et réunie par la Maison au fil du temps ${ }^{5}$. En écho à Pierre Bourdieu (Bourdieu et Delsaut 1975 : 19), on pourrait dire ceci : à mesure que la malle entre dans l'histoire, l'histoire de la malle entre dans la malle. Plus qu'un bien de famille, c'est un reste choyé et continuellement ranimé de ce grand singulier que fut l'ancêtre fondateur. Les malles sont toujours fabriquées dans l'atelier ouvert en 1859 à Asnières, où la «production se donne comme une reproduction» (JeudyBallini 2000: 6). «Reproduire le produit» revient comme un leitmotiv, souligne Monique Jeudy-Ballini, «chercher à s'imiter exactement soi-même par-delà les générations» (ibid.). La Maison Vuitton fait sienne la faculté mimétique, et les vrais clients n'acquièrent rien de moins que des copies originales de la relique. En ce sens, la malle fait fi du temps, elle porte témoignage de la présence du créateur. Métaphoriquement, la malle est à Louis Vuitton ce que le bâton est à saint Pierre.

$\mathrm{Si}$, à l'image des précieuses reliques du Moyen Âge (Cordez 2016), nous disposons pour les malles de notices, de récits d'origine, d'authentification, de consécration, voire de translation, illustrés par la dissémination de copies originales faisant figure d'ersatz, reste la question du miracle, du pouvoir reconnu à l'objet. Une telle capacité revient à la griffe, aux initiales LV imprimées sur la toile monogramme. Dans ce numéro, Brigitte Derlon et Monique Jeudy-Ballini citent sur le sujet l'article pionnier de Pierre Bourdieu et Yvette Delsaut, "Le couturier et sa griffe: contribution à une théorie de la maǵie» (1975), et décrivent le dispositif à l'œuvre dans le champ de la haute couture, qui conduit à la métamorphose de la «qualité sociale» du produit «par la simple apposition d'une griffe ou d'un nom». Qu'est-ce que la griffe? Pour Bourdieu et Delsaut, c'est «un acte symbolique de marquage », «une opération quasi maǵique, manifestée par la signature, qui, par définition, ne peut être opéré qu'en première personne », un acte qui opère la «transsubstantiation» du produit. «Imposer une marque sur le produit, c'est le constituer comme rare, digne d'être recherché, consacré, sacré, légitime [...].» (Bourdieu et Delsaut 1975: 20-22) Le pouvoir singulier de la griffe réside dans la croyance en une valeur produite collectivement, dans ce monde particulier du luxe - Bourdieu utiliserait le mot «champ» - qui fait autorité (ibid. : 23).

Nous reviendrons dans la dernière partie de cette introduction sur ce que Bourdieu et Delsaut appellent l'«appareil de célébration et de consécration», indispensable à l'entretien de la croyance. Relevons pour l'instant un angle mort au sujet de la griffe, assimilée à la signature, en citant cette dernière remarque: «Ce qui fait du Dior, ce n'est pas l'individu biologique, ni la maison Dior, mais le capital de la maison Dior aǵissant sous les espèces d'un individu singulier qui ne peut être que Dior.» (Ibid.: 21) La griffe, reflet de ce capital constamment mobilisé au profit - et offert en l'honneur - du grand singulier, participe de l'histoire, plus ancienne, de l'héraldisation. Sans entrer dans le détail de la destinée de l'écu et du blason, de «cette coutume de marquer d'un signe spécial les biens en tout genre», dira Arnold Van Gennep (1905: 103), avançons plusieurs traits distinctifs, en évoquant d'abord le cadre juridique posé par Bartolo da Sassoferrato vers 1355. Dans son traité De insignis et armis, devenu une référence, Genevière d'Haucourt et Georges Durivault relèvent que «les armes sont, comme les noms, un moyen d'identification ». À cet égard, «armoiries des chevaliers», «seings de notaires » et «marques de fabrique» sont soumises aux «mêmes règles» (Haucourt et Durivault 1992: 28). Le blason est signe de reconnaissance et titre de propriété, transmissible par voie d'hérédité, suivant un cadre légal strictement réǵlementé et concédé reliǵieusement dans nombre de cas (ibid. : 29).

Apposé sur des supports variés, du vêtement à la voiture, le signe, identifié à un détenteur puis à une généalogie familiale - filiation et alliance -, est un droit patrimonial, et, à ce titre, inaliénable. Au blason, sont associés, comme en témoignnent les hérauts, des motifs parfois singuliers, des histoires, des événements glorieux, des prouesses, des registres de consignation, etc. Les dispositions théologico-juridiques qui s'appliquent rappellent celles d'un objet du sacre.

Les enfants nés d'une union non reconnue par la loi, par exemple, ou les actes condamnables se voyaient sanctionnés au travers «d'une dégradation solennelle», "d'une altération infamante des armoiries», punis d'une obligation de "diffamation» (ibid.: 34). Ainsi, le blason est principe et bien moral, officiel et cessible, susceptible de se voir brisé en cas d'infraction grave. Le blason, comme la griffe, est un objet-personne en ce qu'il incarne les qualités d'une personne - ou, par extension, la noblesse d'une filiation. La griffe est assimilable à un nom qui reflète la grandeur d'âme d'une personne, celle du grand singulier à laquelle elle est attachée, et dont bénéficie(nt) son (ses) successeur(s). Mais la griffe est aussi un blason qui parle, un objet autoréférentiel devenant la voix éternelle du créateur, où le récit du jeune Louis Vuitton se confond avec celui, contemporain, de Louis Vuitton. Ornement, le blason relève finalement des technologies de l'enchantement décrites par Gell (1992) au sujet de la proue des canoës utilisés dans les échanges kula.

Le fétiche est également doué de parole. La force d'âme de l'objet à vocation rituelle s'apprécie à l'aune de sa «charge », que les antiquaires ouest-africains se doivent d'atténuer en fonction des circonstances. Un «art de profiler» les pièces, de choisir parmi les états de la chose, suivant la formule retenue par Julien Bondaz. Cet art de faire est indispensable, tant ces intermédiaires ont maille à partir avec leurs voisins ou leurs parents, en raison de la nature de ces artefacts que l'on ne peut jamais complètement convertir en œuvres d'art. Flirtant avec les limites, le marchand de fétiches se voit inlassablement contraint de se défaire des habits du féticheur, et en appelle à la caution morale que représentants coutumiers et religieux peuvent apporter. Derrière les mots et les attitudes, on devine le jeu de combinaisons infinies intéressant la valeur de l'objet, jamais affranchi du jouǵ rituel, ballotté entre ses caractères fétiche et marchand.
5. Voir Olivier Saillard, commissaire de l'exposition : « L'exposition Louis Vuitton en 9 thèmes $»$ [en ligne], RMN, 30 décembre 2015, disponible sur: https://www grandpalais. fr/fr/article/lexpositiondouis$\mathrm{fr} / \mathrm{fr} / \mathrm{article/lexposition-louis-}$ vuitton-en-9-themes (consulté le 08 octobre 2019). 


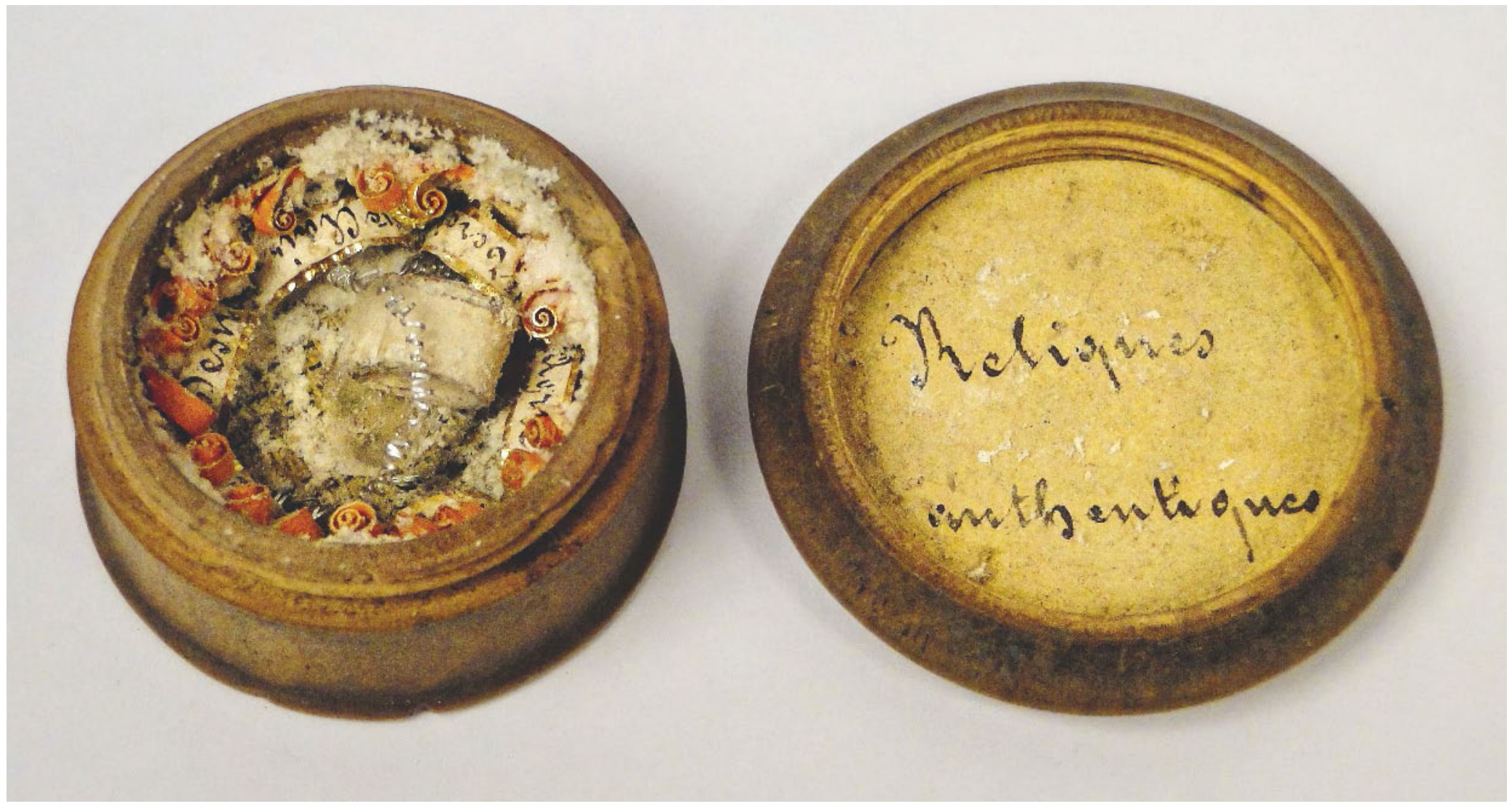

Reliquaire avec paperolles, xxe siècle. Avec l'aimable autorisation de Luc de Laval Antiquités.

Sa nature composite en fait une présence dérangeante et polluante. Il répand un souffle ensorceleur tant sur les lieux qui portent sa trace - la réserve, la boutique, la galerie ou l'espace domestique - que sur les personnes, au travers d'événements malheureux marqués du sceau de la contaǵion. L'objet aǵit, exprime ou «réclame» quelque chose. Pour l'antiquaire, c'est bien le fétiche «qui impose les rèǵles du jeu». C'est l'objet qui choisit le marchand et accepte de «venir vers lui ». Tel est le secret du métier, dévoile l'auteur.

Non loin du fétiche, nous trouvons l'objet coutumier. Honoré Tchatchouang Ngoupeyou en esquisse une typologie dans le cas des chefferies bamiléké de l'ouest du Cameroun. Le conservateur distingue plusieurs ensembles, suivant la manière dont le droit coutumier et l'ordre historique relient certains objets à l'institution royale: fondation, sacre, initiation, règne, économie de prestige, conflit et circulation des biens de valeur. Bloc de pierre, filet de chasse, bracelet, collier, trône, tabouret ou masque participent ainsi des conditions d'exercice de la souveraineté. Le bien coutumier est en cela héritage, amené à endosser les différents visages de l'objet-personne: tour à tour relique, fétiche, objet d'art, quand il n'est pas la réunion des trois. Ces attributs sont autant d'empreintes laissées par les fondateurs et les souverains successifs, gardant ainsi la trace des origines et de la filiation dynastique. Ils portent la marque des événements, des diǵnitaires autorisés à les manipuler, des usaǵes cérémoniels et des soins rituels prodigués au fil des âges. Dans ce régime où le culte des ancêtres fait loi, où les aïeux sont réputés veiller au respect de la coutume, ils sont la voix des défunts, l'incarnation de leur présence par-delà le temps. Le bien coutumier est «compliqué», chargé à l'instar du fétiche. Même entreposé de longue date dans un musée occidental, il ne perd rien de sa "puissance», dont on mesure l'étendue à l'aune du dispositif de conservation et de protection mis en œuvre. L'objet coutumier institue, rayonne, menace et protège.

«Ne me vendez pas. Si tu me vends, tu vas devenir fou », dit un jour un masque à l'antiquaire. L'avertissement fait écho aux récits de vol de reliques, qui ne faisaient qu'en confirmer la valeur et le pouvoir. Dans ce marché soutenu du $\mathrm{X}^{\mathrm{e}}$ au XIV $\mathrm{X}^{\mathrm{e}}$ siècle, la circulation des restes consistait notamment en larcins.

Patrick Geary évoque des affaires impliquant des voleurs obligés de composer avec l'objet convoité, afin que ce dernier accepte de se laisser emporter par le maraudeur ou bénéficie d'une vénération plus grande encore au sein de sa future communauté d'adoption (Geary 1986: 187). On négocie avec le fétiche comme on négocie avec la relique. Dans les deux cas, le rappel à l'ordre, l'infraction ou le recours à la ruse - choisis comme procédés narratifs - ne font que renforcer leur caractère insubstituable. Si le masque ou la relique ne résistaient pas, leur classe exceptionnelle ne manquerait pas d'être discutée.

Ce régime des personnes, au fondement de leur qualité, appelle sans cesse un «travail de particularisation» (Heinich 1993: 30), prenant modèle sur l'unicité absolue reconnue aux personnes et à leur profil créateur, artiste, saint, féticheur, monarque ou autorité coutumière. La notice, «état civil » établi pour l'objet précieux, en est la siǵnature. Elle distinguue les œuvres d'art et les reliques, individualise les récits de fondation ou de découverte, d'usage et d'acquisition dans le cas des fétiches et des attributs coutumiers. 
Ces choses agissent "parce qu'on a aǵi sur [elles]», suǵgère Gell au sujet des fétiches à clous (2009: 75). Nous dirions qu'elles aǵissent parce qu'on ne cesse d'aǵir sur elles. Nos objets précieux peuvent s'honorer d'avoir un nom, un âge, une origine, une filiation, une trajectoire, des qualités extraordinaires, autant de traits distinctifs qui, comme pour les personnes, ne sont pas réductibles à ce qu'ils sont vraiment. Le travail de particularisation vise à en faire (toujours) plus que la somme de leurs composantes (Heinich 1993 : 37). En ce sens, la théorie anthropologique de l'art proposée par Gell s'apparente à une théorie anthropologique des rapports à l'objet devenu insubstituable.

Un objet-personne peut-il fuir sa destinée? Loin de l'attachement qui fait sa singularité, l'objet serait dès lors recherché ou choisi pour sa rareté et son ancienneté, suggère Nathalie Heinich.

L'anthropologue aurait tendance à nuancer la proposition en considérant que la longévité promise à la chose est consubstantielle à sa nature. L'ancienneté désigne à la fois l'état de ce qui est ancien et la continuité dans le temps. La continuité s'exprime dans une histoire, au travers de liens tissés entre des épisodes, des individus et des usages précisément, ceux auxquels renvoient les doudous, les reliques ou les masques dans l'imaǵinaire individuel et collectif. Pour paraphraser notre collègue, le temps ne fait qu'ajouter à «ces valeurs ajoutées » qui sécrètent l'attachement. Bourdieu identifie, dans l'échange différé des biens de prestige, l'opération qu'il désigne par «manipulation de la durée», indissociable à ses yeux du bien-fondé des dons (2000 : 339-340). Dans notre cas, cette opération vise à aǵǵlomérer, par addition et mélange, les états de la chose. L'objet-personne «complet», si bien illustré par La Joconde, incarne le summum du précieux. La peluche ou le teddy-bear ne cessent jamais vraiment d'être une personne, comme les masques des fétiches. Même ordinaire ou «petit rien », l'objet-personne ne peut échapper à son sort, assuré de se bonifier avec le temps. Le doudou ancien continuera à montrer ses visages de double vital et de fétiche, sous les traits de la rareté et la forme d'une collection. À cette même question, l'antiquaire ouest-africain répondrait sans l'ombre d'un doute que ses fétiches, même convertis en œuvres d'art, continuent de porter la marque indélébile du fétiche.

\section{CONTINUITÉ ET DISCONTINUITÉ}

L'emblème du Roi-Soleil, visible sur les gardecorps des fenêtres du premier étage, est à considérer à l'aune de deux usaǵes caractéristiques de la figure et du règne du souverain que consacre l'inauguration de la Maison Louis Vuitton. Le premier est fondé sur un rapport de similitude établi entre le monument restauré et le château de Versailles (et son glorieux commanditaire); le second est illustré par deux installations artistiques: l'astre solaire irradiant la façade de ses rayons, prolongée jusqu'à fin décembre 2018, et une statue de grande dimension dressée au pied de l'escalier, dont le style et les motifs sont empruntés à l'aquarelle du costume que le jeune Louis revêtit pour la représentation du ballet La Nuit en 1653 , où le dauphin apparut pour la première fois «habillé

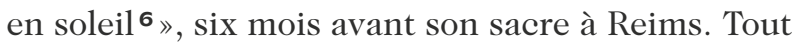
revêtu d'or, qui est ce personnage représenté? Louis XIV jeune ou Louis Vuitton jeune? Ou quelqu'un d'autre? Un indice nous est donné par les initiales « $L V$ » figurant sur le fermoir du ceinturon et la boucle des souliers.

Dans ce dernier cas, la griffe s'est substituée à l'emblème du soleil. Cette création, un temps prévue pour l'inauguration, fait dorénavant partie du décor. Ainsi, la communication du groupe et certains choix artistiques s'attachent à illustrer ce thème bien connu du corps politique et sacré du roi. Le retour aux origines pour Vuitton, signifié par l'ouverture de la Maison non loin du magasin du fondateur, se mêle avec la naissance de la place Vendôme voulue par Louis XIV. Deux illustres fondateurs invités à dialoguer, malǵré les deux siècles qui les séparent. Notons, au passage, ces dates anniversaires: 1654 fut l'année du sacre de Louis XIV, 1854 celle de l'ouverture de la boutique de Louis Vuitton.

Dans son ouvrage Ce que nous voyons, ce qui nous regarde, Georges Didi-Huberman intitule son premier chapitre: «L'évitement du vide: croyance et tautologie» (2014 [1992] : 17-25). Face au tombeau, Didi-Huberman caractérise deux expériences possibles du voir : celle qui consiste en un exercice de tautologie, à ne voir dans le tombeau qu'un volume, qu'une forme particulière modelée ou construite dans un matériau particulier, «en récusant les latences de l'objet», «le travail du temps ou de la métamorphose de l'objet, le travail de la mémoire - ou de la hantise - dans le regard» (ibid.: 19). Une expérience qui ne fait que dissiper l'aura de l'objet. L'autre relève d'un exercice de la croyance, qui s'impose comme «une vérité superlative et invocante», en postulant un "quelque chose d'Autre" qui fait revivre tout cela et lui donne un sens, téléologique et métaphysique» (ibid.: 21). S'il discute, voire condamne ces deux expériences du voir, il n'en reste pas moins qu'elles composent les «deux faces de la monnaie», témoins en cela de l'inéluctable scission du voir. Lidée du «vide» ou du «rien», corrélative de celle de continuum, revient à interroger sur le plan anthropologique les rapports à la pérennité et à l'universalité, en ce qu'ils concilient continuité et discontinuité. Suivant l'argument développé par Appadurai (1986: 41-56), deux substrats indivisibles sont dès lors à considérer: l'un mythologique, l'autre rituel.

L'appel du pied lancé à la royauté dans la promotion de la Maison Vuitton suǵgère quelques mots sur l'institution du trésor royal. Yann Potin l'a démontré pour la fin du Moyen Âge: les biens de valeur mis au secret constituent une réserve qui satisfait aux besoins du régime, en matière d'héritage, d'alliance, de liturgiie, d'autorité et de finance. Ces biens sont enchâssés dans des sphères potentiellement contradictoires : le merveilleux, la croyance, la transmission, l'échange restreint, l'activité politique et cérémonielle, la réserve monétaire et le recyclage. Le terme «trésor», préciset-il, «désiǵne [...] les lieux où se concentre la valeur sous toutes ses formes» (Potin 2004: 53). La valeur sous toutes ses formes, comme des visages de l'attachement,
6. Voir « Costumes du ballet intitulé La Nuit, représenté à la cour en 1653 , dans lequel Louis XIV figura habillé en soleil », dessin [s.d], BNF Gallica. 
où le beau se trouve indissociablement lié au merveilleux, en ce que le trésor abrite antiquités et exotica, médecines et amulettes. Avec les pierres précieuses, l'attachement est deliciae, mais aussi croyance avec la présence des reliques. Le dispositif se déploie en circuit fermé, et l'usage desdits biens obéit à des formes ritualisées. Par sa nature hors du commun, le roi consacre tout ce qu'il possède, tout ce qu'il touche, tout ce qu'il porte. La transsubstantiation opère en vertu de ces trois composantes: un objet estimé ou inestimable, un lieu gardé et tenu secret, et un médiateur divinisé. Honoré Tchatchouang nous rappelle à cet égard que le cas de la royauté européenne n'est pas unique. Dans les chefferies bamiléké, le souverain confère un supplément d'âme aux objets qui l'entourent. Ils sont en cela frappés d'interdit.

Louis Vuitton n'est pas roi, mais l'image vivante qui nous est transmise est celle d'un fondateur idéalisé sur le modèle de Louis XIV. Ou, pour le dire autrement, Versailles et son illustre souverain sont aussi éternels que Louis Vuitton et sa maison. La consécration de la Maison Vuitton, la raison d'être du récit qui s'y rattache et des œuvres plastiques présentées, repose sur une opération que Bourdieu a nommée «rite d'institution». Sur un mode solennel, cette action rend licite un déplacement de la «liǵne» qui sépare «un avant» et «un après» en rendant «légitime» un fait ou une histoire (Bourdieu 1982: 58), en établissant, dans notre cas, un fondateur et son héritage dans un monument au passé (re)composé. L'inauguration constitue ce temps cérémoniel qui autorise la célébration de ce récit, au travers notamment de la soirée d'ouverture animée par des personnalités des arts et du spectacle, et de la couverture médiatique de l'événement, où magazines spécialisés et quotidiens nationaux chantent à travers le monde la vie glorieuse de la marque. Brigitte Derlon et Monique Jeudy-Ballini reviennent ici sur le profil de ces acteurs qui composent et attestent ces versions enrichies du mythe.

L'efficacité symbolique des rites d'institution, avance Bourdieu, réside dans «le pouvoir qui leur appartient d'aǵir sur le réel en aǵissant sur la représentation du réel» (ibid. : 59). Pour l'auteur, instituer est synonyme de consacrer, en vertu d'un appel à un tiers légitime, un représentant qui confère un sens à l'acte (Abélès 1990: 245-246). Observons à cet égard la statue du personnage en costume de ballet, qui arbore la griffe sur la boucle de ses souliers en lieu et place de l'emblème du soleil. Si Louis XIV joue le rôle de la divinité, alors cette œuvre relève d'un acte - d'un geste artistique - de déification. À l'image de l'empereur romain, l'apothéose est couronnée par sa représentation sur une pièce ou une médaille. L'acte de déification repose sur la personne du monarque, une figure, qui, métaphoriquement, prend en charge la fonction de délégation et autorise l'apothéose de Louis Vuitton.

Le luxe produit et reproduit de la continuité.

L'industrie qui le porte ne peut y échapper en raison d'un trait essentiel: la superfluité propre à ce régime de consommation (Abélès 2018: 35). Un penchant inconvenant, tantôt jugé contraire aux bonnes mœurs et au bien-être collectif, tantôt signne de progrès et facteur de vitalité économique. Le désir inassouvi, ou le désir futile et incessant, inspire de nouvelles réflexions au XVIII ${ }^{\mathrm{e}}$ siècle, au moment où le luxe se voit associé à l'intensification des échanges avec le lointain. L'Orient fascine, les Compaǵnies des Indes se développent, pagodes, bronzes et céramiques de Chine, porcelaines du Japon, «morceaux d'histoire naturelle», «agathes et [...] toutes marchandises curieuses et étrangères » se voient déclinés sur différents supports, de la tapisserie au mobilier (Verlet 1958 : 16-17). Le parallèle avec les deliciae terrestres de la haute société romaine du ${ }^{\mathrm{er}}$ siècle est manifeste.

Le luxe a partie liée avec l'altérité (Abélès 2018: 79), et révèle ce ressort puissant à l'œuvre également dans la thésaurisation, dans cet assemblage de signes qui condensent - et renouvellent - des «représentations du passé, de l'au-delà, de l'ailleurs [...] » (Marin 1986: 12). Pensons à ces œufs d'autruche, noix de coco et coquilles de mollusques montés en reliquaire dans le sillage des croisades (Cordez 2016: 145-158), ou, plus proches de nous, aux projections imaginaires décrites dans le cas des collectionneurs (voir Brigitte Derlon et Monique Jeudy-Ballini dans ce numéro). La quête de l'ailleurs inspire le désir de posséder le "Tout-Autre» pour reprendre le mot d'André Malraux (1953 [1951] : 512). En ce sens, superfluité et accumulation pourraient bien relever d'un même motif, où l'un forme le pendant de l'autre. Cette petite phrase de Pline, citée par Pierre Schneider, résume bien l'idée: «Nous buvons dans une multitude de gemmes, nous composons des coupes d'émeraudes entremêlées et nous nous plaisons à tenir l'Inde entre nos mains pour nous procurer l'ivresse.»

Le caractère ambivalent du désir du «Tout-Autre» pose cependant de singuliers problèmes de sens. La création, la circulation ou la sauvegarde des objetspersonnes doivent en effet se justifier. À l'instar de la Maison Vuitton, un récit est attendu ainsi qu'un acte particulier qui le rend crédible en garantissant sa valeur performative. Les contributions réunies dans ce numéro illustrent davantage le premier versant que le second. Dans la plupart des cas cités, le récit magnifie la distance géographique. Les collectionneurs invoquent «les antipodes» pour valoriser la provenance de certaines pièces. Un motif du lointain, qui se confond avec celui du passé, pour exprimer tant le point de départ que l'ascendance. De la même façon, l'origine mythique des biens en coquillage mélanésiens est indissociable de leur nature étrangère. Pline insiste aussi sur ce thème, allant jusqu'à l'exaltation de contrées singulières, où la nature confine au sublime. Par un anachronisme, le dessein encyclopédique de Pline rejoint celui de Louis Vuitton. Dans les deux cas, il s'agit de donner sens à un tout, de rétablir la continuité malgré les discontinuités en proposant une synthèse cohérente de l'universel. Les antiquaires ouest-africains, quant à eux, usent des figures du «baroudeur» et de «l'aventurier» pour prouver leur expérience et leur expertise. Ces références à la distance et à l'expédition renforcent le thème central de la découverte du fétiche, constituant la trame du récit.

Conjointement, une certaine discrétion entoure leurs circuits d'approvisionnement, note Julien Bondaz. Un mélange de réserve, de retenue ou de pudeur propre 


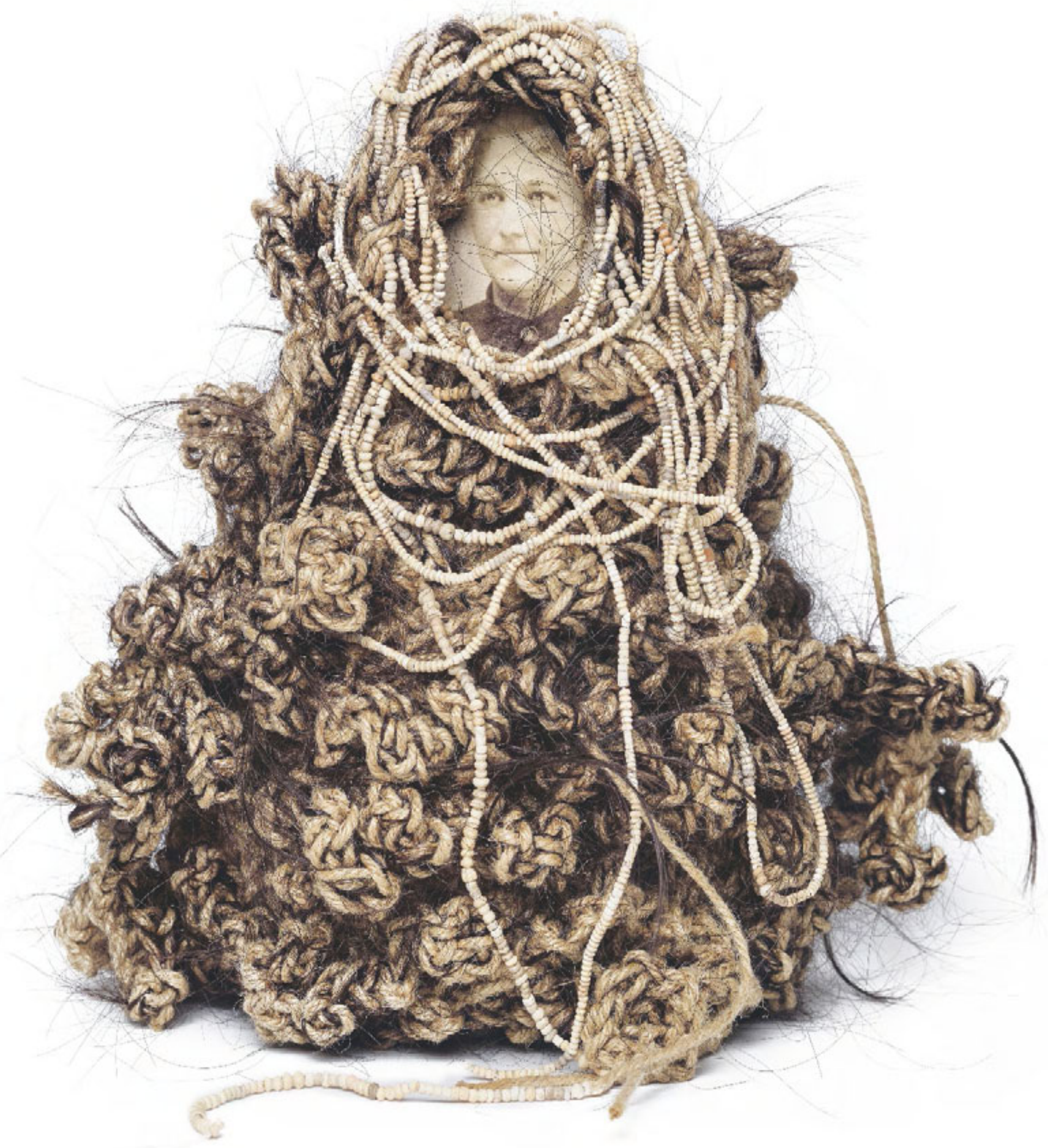

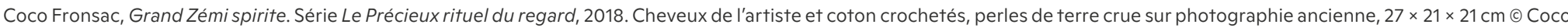
Fronsac. Photographie (c) Christophe Lebedinsky. 


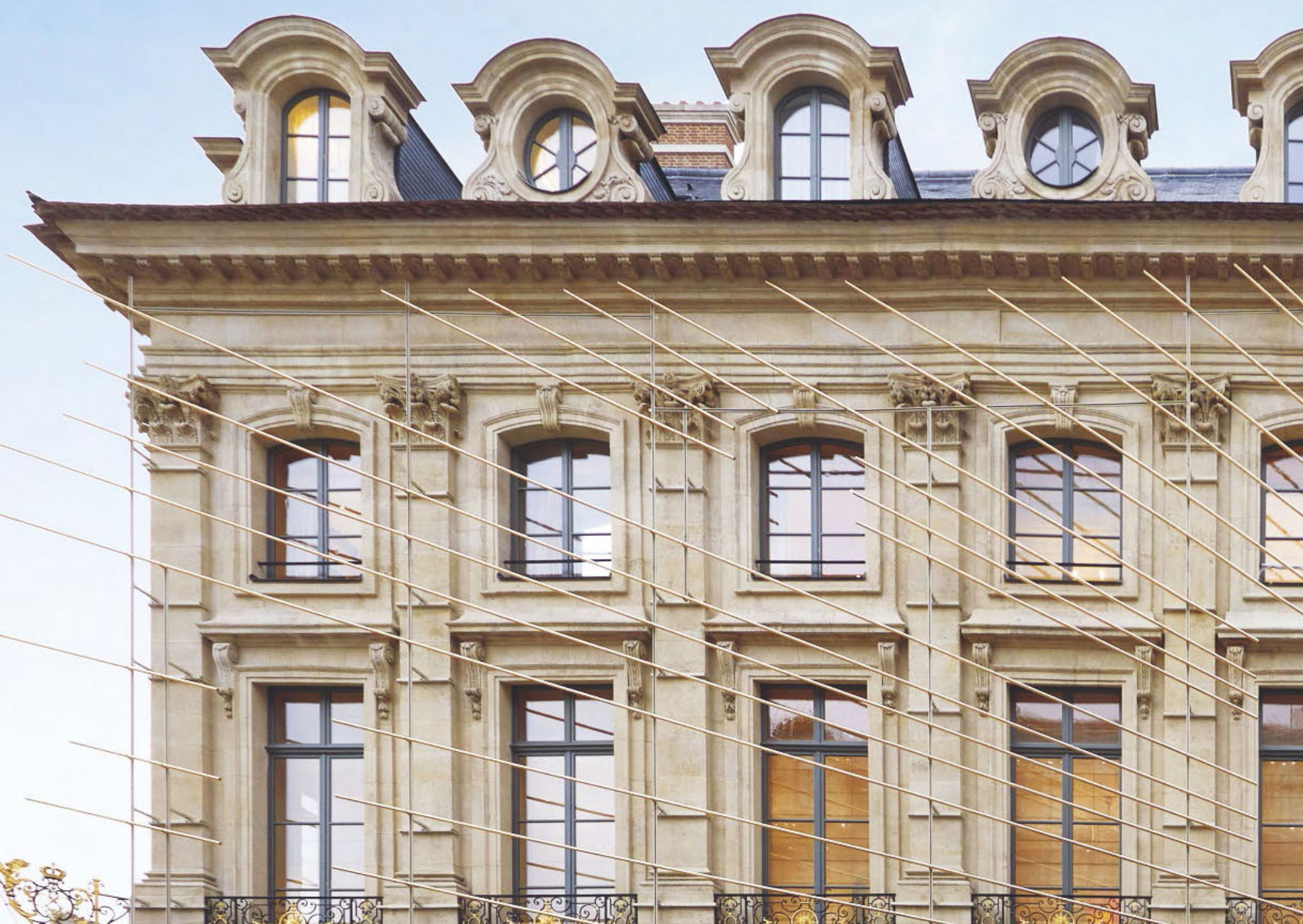

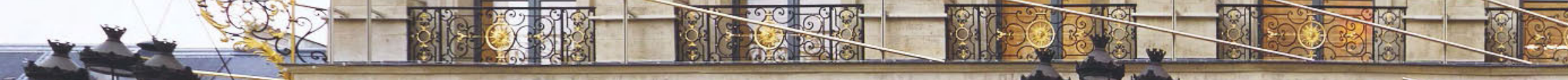

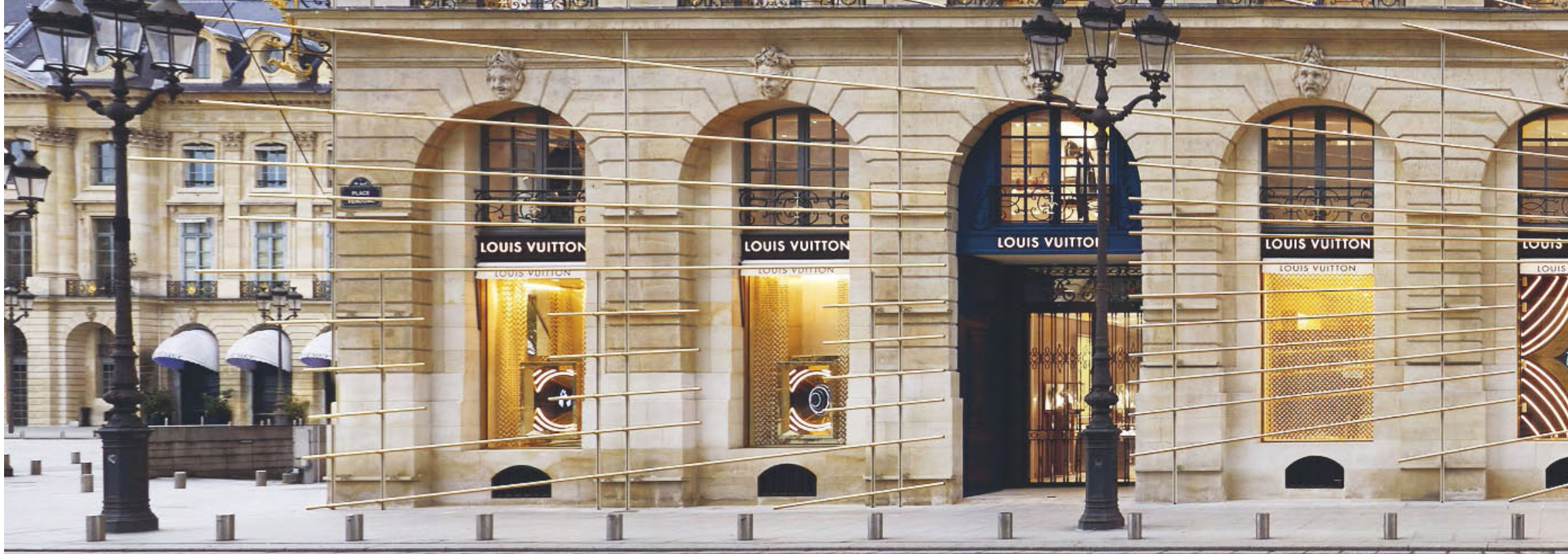




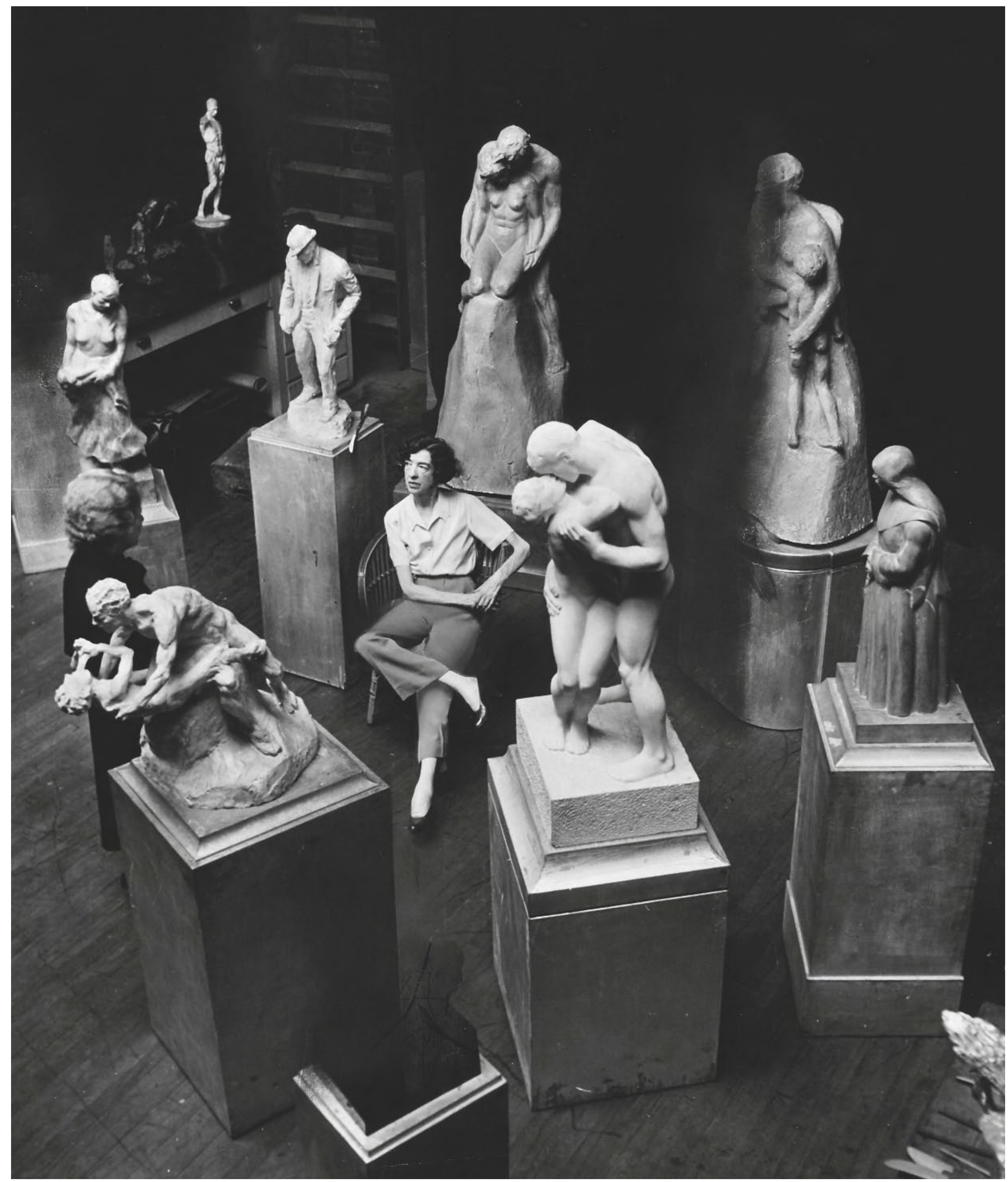

Herbert Gehr, Juliana R. Force, directrice du Whitney Museum de New York, discutant avec la mécène et sculptrice Gertrude Vanderbilt Whitney, 1939. Photo Herbert Gehr/The LIFE Images Collection via Getty Images/Getty Images. 
à nourrir de mystères l'objet et à offrir une expérience de la révélation, qui s'avère empruntée au modèle initiatique $^{7}$. La distance, ou le parcours tumultueux d'un bien, depuis son point de départ jusqu'à son point d'arrivée, a inspiré à Appadurai des réflexions judicieuses: le mystère entretenu va de pair avec le savoir disponible à son sujet et les conditions d'accès à ce savoir. L'anthropologue s'intéresse en particulier aux aspects de la connaissance liés aux itinéraires lonǵs, où sont en jeu échanges et transferts culturels. Dans ces situations, souligne-t-il, «la tension entre connaissance et ignorance devient un déterminant critique des flux de marchandises » (Warnier 2009: 45).

Les caractéristiques du bazar marocain relevées par Glifford Geertz en matière d'information sur les produits en font un cas d'école. Le savoir partiel a pour corollaire la quête de sens, et Appadurai élargoit la portée de ce rapport de symétrie à toute forme d'échange de biens non standardisés. Ce besoin s'exprime en termes d'exclusivité, d'authenticité et d'expertise (Appadurai 1986: 49). Le travail d'authentification desdits biens s'appuie sur des critères rationnels et d'autres qui le sont moins. Pour les objetspersonnes, comme le fétiche ou la relique, l'histoire, l'itinéraire et l'usage ou le récit des «carrières" prennent beaucoup d'importance. D'un autre côté, l'objet est personne parce qu'il est victime de son origine lointaine. La proposition d'Appadurai intéressant le sens donné aux flux de marchandise s'avère pertinente dans notre cas. Des mythologies naissent en raison d'une double incertitude: la première liée à la visibilité inégale, voire à l'absence de visibilité sur les circulations à longue distance, la seconde à «l'aliénation mutuelle des producteurs, négociants et consommateurs» (ibid. : 53). À mesure que progressent les discontinuités d'ordre social, spatial et temporel, la mythopraxis, pour reprendre le terme de Marshall Sahlins, engendre de la continuité. Les cultes du cargo que pointe Appadurai révèlent une logique plus complexe. Né dans le Pacifique au moment de la rencontre avec les Européens, ce messianisme annonce l'apparition d'objets-personnes inédits, fruit d'un processus de «fétichisation » à l'envers. Les biens représentatifs servant à la pratique rituelle donnent corps et réalité au transfert et à l'assimilation des «formes sociales européennes» jugées «puissantes». Le choix se porte sur les objets qui, aux yeux des adeptes, illustrent le plus ouvertement l'ordre hiérarchique des Blanes (ibid.: 57). Ici, le propre de l'objet-personne est de garantir, sous de nouveaux auspices, la permanence des discontinuités. À l'instar de l'inéluctable scission du voir, des états agǵlomérés de la chose, continuité et discontinuité s'appellent et se répondent mutuellement, en laissant leur empreinte commune ou leurs reflets confondus sur l'objet-personne.

La mémoire fraǵmentaire, l'iǵnorance ou la connaissance partielle de la trajectoire de ces biens, voire l'éniǵme de l'origine, rendues tangibles par l'éloignement, dans l'espace et dans le temps, se trouvent compensées par la croyance. La longue distance, l'ancienneté, la complexité de la chaîne opératoire qui leur est associée, leur relative rareté, appellent invariablement une rhétorique de la découverte sinon de la révélation. En matière de biens symboliques, la production de la croyance relève d'un processus que Bourdieu et Delsaut nomment «alchimie sociale». Le principe de cette transformation presque miraculeuse repose sur une méconnaissance fondamentale: aucun des acteurs impliqués ne maîtrise l'ensemble du dispositif qui concourt à faire d'un objet un précieux aucun d'entre eux, qui plus est, ne mesure véritablement les effets de sa propre contribution à la production de l'exceptionnalité (voir Brigitte Derlon et Monique Jeudy-Ballini dans ce numéro). La croyance reposerait ainsi sur une sorte d'évidence: celle de l'incomplétude du savoir associé à l'objet-personne. L'adhésion comble les trous ou les vides, l'assentiment confère à l'objet un surplus d'âme, qui l'érige en un tout cohérent. De là, tirons-nous, peut-être, l'élixir de l'authenticité.

Avant de laisser la parole aux auteurs, revenons à notre énoncé tenant lieu d'hypothèse de travail. Au travers des cas décrits, nos objets se voient-il dotés d'une sacralité, consubstantielle à la valeur qui leur est assignée? À tout le moins, nous observons des mises en situation codifiées, dictées par des besoins qui se muent bien souvent en protocole, des troubles confus, passagers ou durables, ressentis à leur endroit, et des registres de discours préférentiels leur permettant de revêtir un caractère de solennité et de grandeur. L'idolâtrie témoiǵne à cet égard de la condition humaine de certains objets, où l'attachement, l'aversion ou l'étrangeté sécrètent la croyance. De même, insistons sur l'extraordinaire mythologie qui justifie leur raison d'être. Transposée en soins particuliers, l'allégorie rend le précieux dépositaire d'une vérité. Si tabous et interdits se devinent derrière les usaǵes, aucun corpus présenté dans ce numéro ne permet de dessiner les contours d'un périmètre rendu inviolable. Mais, comme il est souligné à juste titre, on pourrait mieux encore établir la gamme du précieux sur la base de ces contraintes et de leur nature.
7. Suivant I'hypothèse émise par Christopher Steiner dans son étude du marché des masques africains, et que reprend Julien Bondaz: « The Secret of the Masks: On the Social Construction of Power and Desire in the African Art Market ", in Silvia Forni et Christopher Steiner (dir.) Africa in the Market:

Twentieth-century Artfrom Amrad African Art Collection, Toronto, Royal Ontario Museum, 2015 : 146-163.

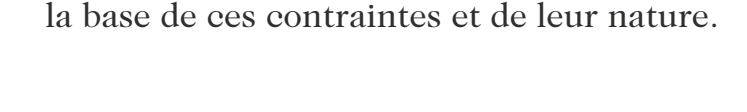

(




\section{Bibliographie}

\section{Abélès, Marc}

1990 «Mises en scène et rituels politiques. Une approche critique », Hermès 8-9:

241-259.

2018 Un ethnologue au pays du luxe. Paris, Odile Jacob.

\section{Appadurai, Arjun}

1986 «Introduction: Commodities and the Politics of Value»,

in Arjun Appadurai (dir.)

The Social Life of Things

Commodities in Cultural

Perspective. Cambridge,

Cambridge University Press :

3-63.

\section{Baudrillard, Jean}

2015 [1968] Le Système

des objets. Paris, Gallimard.

\section{Bohannan, Paul} et Bohannan, Laura

1968 Tiv Economy. Evanston, Northwestern University Press.

\section{Bonnot, Thierry}

2014 L'Attachement aux choses. Paris, CNRS Éditions.

\section{Bourdieu, Pierre}

1982 «Les rites comme actes d'institution », Actes de la recherche en sciences sociales $43: 58-63$.

2000 [1972] Esquisse d'une théorie de la pratique, précéde de trois études d'ethnologie kabyle. Paris, Seuil.

\section{Bourdieu, Pierre} et Delsaut, Yvette

1975 «Le couturier et sa griffe contribution à une théorie de la maǵie », Actes de la recherche en sciences sociales 1: 7-36.

\section{Cocteau, Jean}

1959 Poésie critique. Paris, Gallimard.

\section{Cordez, Philippe}

2016 Trésor, mémoire, merveilles: les objets des églises au Moyen Âge. Paris, Editions de l'EHESS.

\section{Dassié, Véronique}

2010 Objets d'affection une ethnologie de l'intime. Paris, Éditions du CTHS.

\section{Derlon, Brigitte} et Jeudy-Ballini, Monique 2008 La Passion de l'art primitif: enquête sur les collectionneurs.

Paris, Gallimard.

\section{Didi-Huberman, Georges}

2014 [1992] Ce que nous voyons, ce qui nous regarde. Paris, Minuit.

\section{Fabre, Danie}

2013 «Le patrimoine porté par l'émotion», in Daniel Fabre (dir.), Émotions patrimoniales. Paris, Éditions de la MSH: 13-98.

2015 «Monument, patrimoine et politiques du sacré. Conquérir Vezelay», communication présentée dans le cadre du colloque «Les patrimoines en recherche(s) d'avenir», Paris, BNF, 24 septembre.

\section{Foucault, Michel}

2001 [1975] Surveiller et

punir: naissance de la prison. Paris, Gallimard

\section{Freedberg, David}

1998 [1989] Le Pouvoir des images, trad. de l'anglais par Alix Girod.

Paris, Gérard Monfort.

\section{Geary, Patrick}

1986 «Sacred Commodities: The Circulation of Medieval Relies », in Arjun Appadurai (dir.), The Social Life of Things: Commodities in Cultural Perspective. Cambridge, Cambridge University Press : 169-191.

\section{Gell, Alfred}

1992 «The Technology of Enchantment and the Enchantment of Technology », in Jeremy Coote et Anthony Shelton (dir.), Anthropology, Art and Aesthetics.

Oxford, New York, Oxford

University Press : 40-63.

2009 [1998] L'Art et ses agents, une théorie anthropologique, trad. de l'anglais par Sophie et Olivier Renaut. Dijon, Les presses du réel.

\section{Godelier, Maurice}

1996 L'Énigme du don Paris, Fayard.

\section{Gregory, Chris A.}

1982 Gifts and Commodities. London/New York, Academic Press.

Haucourt (d'), Geneviève et Durivault, Georges 1992 [1960] Le Blason.

Paris, Presses universiaires de France.

\section{Heinich, Nathalie}

1991 La Gloire de Van Gogh essai d'anthropologie de l'admiration. Paris, Minuit.

1993 «Les objets-personnes. Fétiches, reliques et œuvres d'art », Sociologie de l'art 6 : 25-56.

2017 Des valeurs : une approche sociologique. Paris, Gallimard.

Jeudy-Ballini, Monique

2000 «Déficitaires et ǵlorieux Limaǵinaire du luxe et de l'authentique chez les salariés d'une entreprise de maroquinerie», Terrain 35 : 141-154.

\section{Kott, Christina}

2013 «Guerre et patrimoine.

L"Exposition des œuvres

d'art mutilées" de 1916 », in

Daniel Fabre (dir.), Émotions patrimoniales. Paris, Éditions de la MSH : 119-145.

\section{Malraux, André}

1953 [1951] Les Voix du silence. Paris, Gallimard.

Marin, Louis

1986 «Fraǵments d'histoires de musées ", Cahiers du Musée national d'art moderne 17-18: 8-17.

\section{Mauss, Marcel}

2012 [1950] «Essai sur le don. Forme et raison de l'échange dans les sociétés archaïques», in Marcel Mauss, Sociologie et anthropologie. Paris, Presses universiaires de France: 143-279.

\section{Meillassoux, Claude}

1960 «Essai d'interprétation du phénomène économique dans les sociétés traditionnelles d'auto-subsistance», Cahiers d'Études africaines 1-4: 38-67.

\section{Pietz, William}

2005 Le Fétiche: généalogie d'un problème, trad. de l'anǵlais par Aude Pivin.

Paris, Karǵo-L'Éclat.

\section{Polanyi, Karl}

1957 «The Economy as Instituted Process », in Karl Polanyi et al. (dir.), Trade and Market in the Early Empires: Economies in History and Theory. Chicago, Henry Regnery Company : 243-270.

\section{Potin, Yann}

2004 «Entre trésor sacré et vaisselle du prince. Le roi médiéval est-il un collectionneur?», Hypothèses 1: 45-56.

\section{Sahlins, Marshall}

1976 [1972] Âge de pierre, âge d'abondance: l'économie des sociétés primitives. Paris, Gallimard.

\section{Simmel, Georg}

2014 [1911] Philosophie de l'argent. Paris, Presses universiaires de France.

\section{Sombart, Werner}

1922 Luxus und Kapitalismus. Munich, Duncker \& Humblot.

\section{Van Gennep, Arnold}

1905 «Notes sur l'héraldisation de la marque de propriété et les origines du blason", Bulletins et Mémoires de la Société d'anthropologie de Paris 5-6: 103-112.

\section{Verlet, Pierre}

1958 «Le commerce des objets d'art et les marchands merciers à Paris au XVIII ${ }^{\mathrm{e}}$ siècle ", Annales. Histoire, Sciences Sociales 13 (1) : 10-29.
Vuitton, Louis

2017 Maison Louis Vuitton Vendôme, dossier de presse.

\section{Warnier, Jean-Pierre}

1985 Échanges, développement et hiérarchies dans le Bamenda précolonial (Cameroun) Stuttgart, Franz Steiner Verlaǵ Wiesbaden.

2009 «Arjun Appadurai Les marchandises et les politiques de la valeur», Sociétés politiques comparées 11: 1-70.

\section{Weiner, Annette B.}

1992 Inalienable Possessions The Paradox of KeepingWhile-Giving. Berkeley/ Los Angeles/Oxford, University of California Press.

\section{Ci-contre et ouverture}

Anonyme espagnol, La Dame auxpensées, $x v^{e}$ siècle détail), huile sur bois, $27 \times 36 \mathrm{~cm}$. Musée du Louvre, Photo $\odot$ RMN-Grand Palais (musée du Louvre) / Franck Raux. 


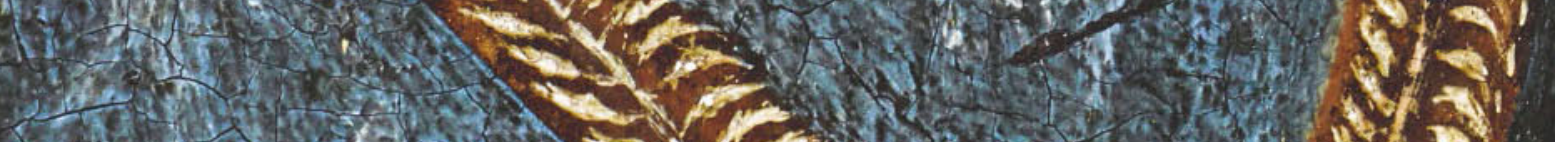

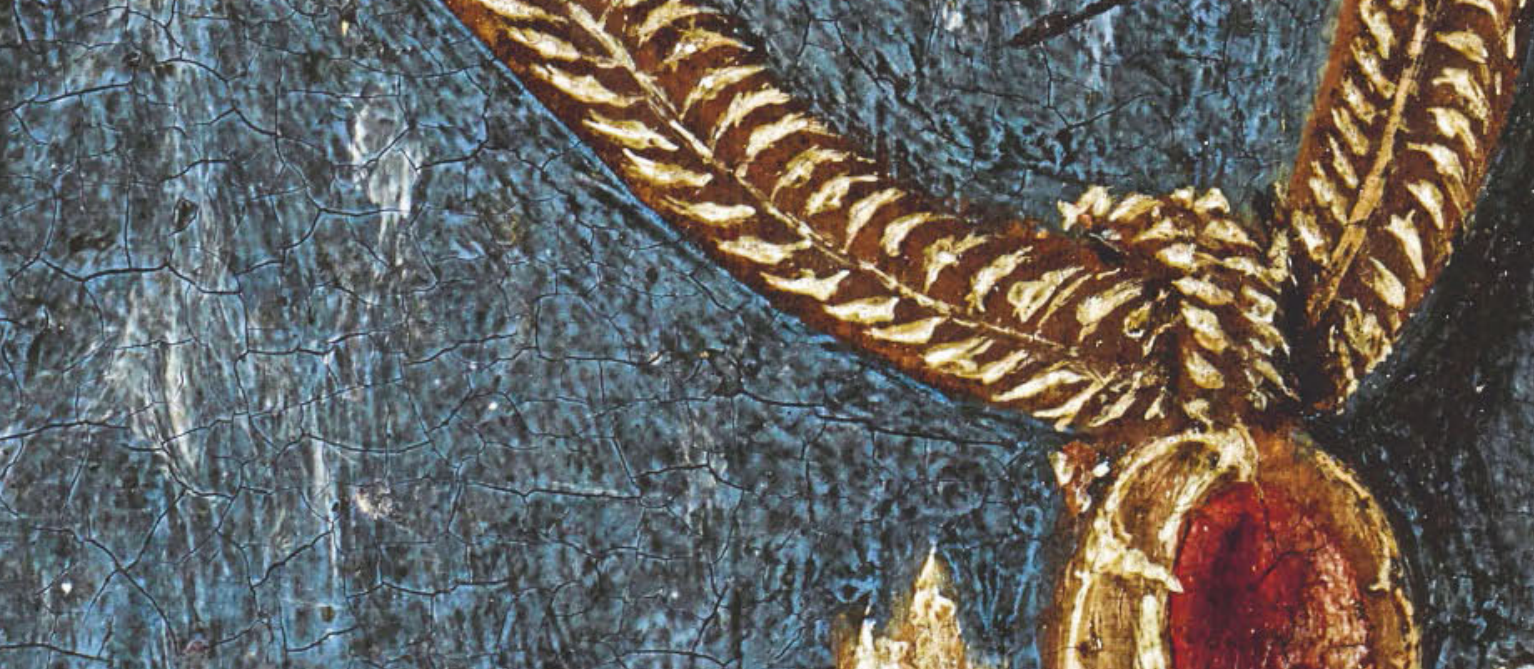

N3)

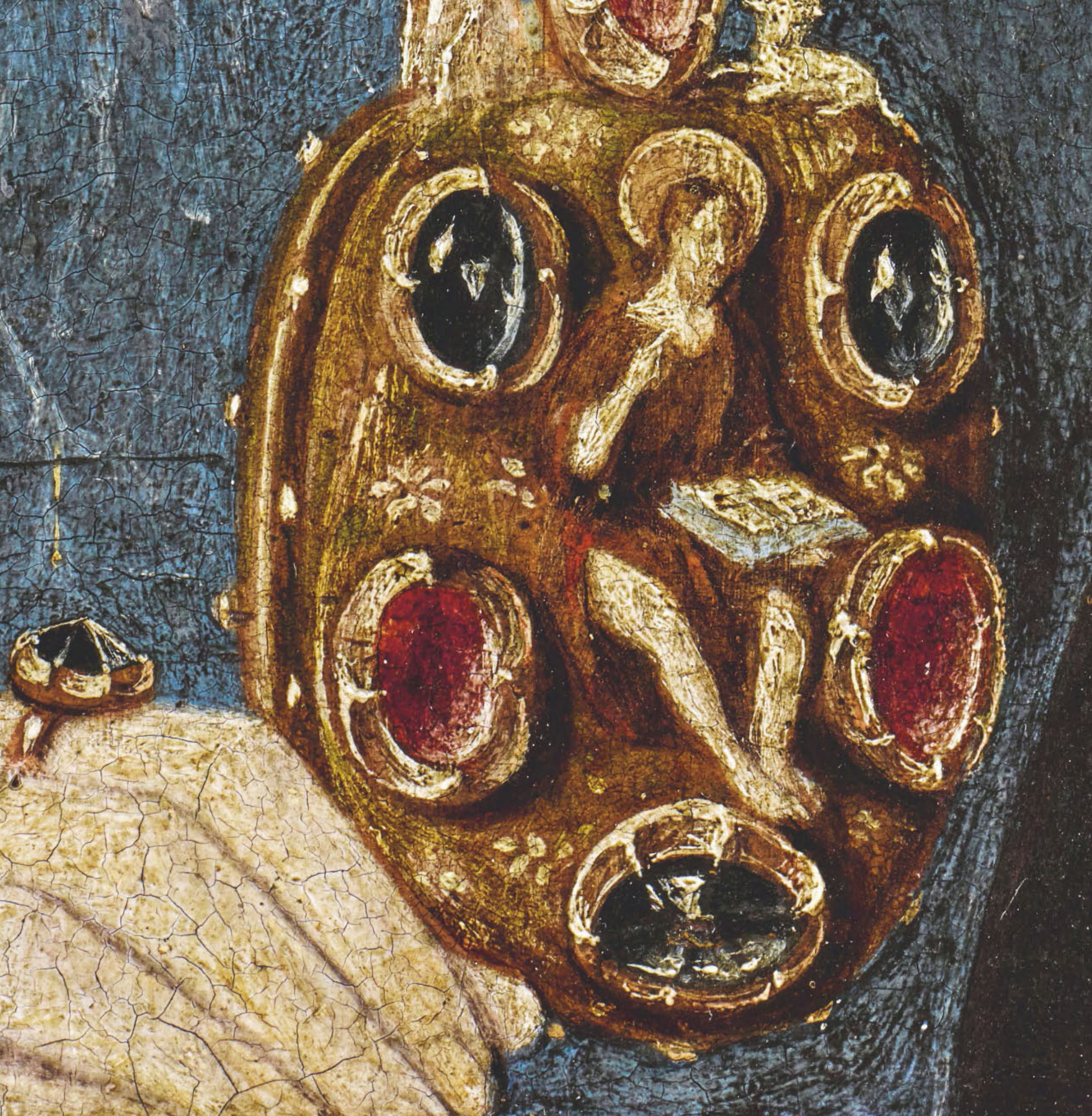

